Vol. 00, No. 00, 1-21, 00 Month 20xx

\title{
An radial basis function scheme for option pricing in exponential Lévy models
}

\author{
RAYMOND BRUMMELHUIS* \& RON T.L. CHAN ${ }^{\dagger}$ \\ * Department of Economics, Mathematics and Statistics, Birkbeck, University of London, Malet Street, London WC1E \\ $7 H X, U K,{ }^{\dagger}$ UEL Royal Docks Business School University of East London, Docklands Campus, 4-6 University Way, \\ London E16 2RD
}

(Received 00 Month 20xx; final version received 00 Month 20xx)

\begin{abstract}
We use Radial Basis Function (RBF) interpolation to price options in exponential Lévy models by numerically solving the fundamental pricing PIDE. Our RBF scheme can handle arbitrary singularities of the Lévy measure in 0 without introducing further approximations, making it simpler to implement than competing methods. In numerical experiments using processes from the CGMY-KoBoL class, the scheme is found to be second order convergent in the number of interpolation points, including for processes of unbounded variation.
\end{abstract}

KeY Words: Option pricing in exponential Lévy models, CGMY-KoBol and VG processes, Partial Integro-Differential Equations (PIDE), Radial Basis Function interpolation, Multi-quadrics.

\section{Introduction}

This paper investigates a radial basis function-, or RBF, -scheme for numerically solving the derivative pricing equation of exponential Lévy models. The scheme is simple to implement, and is capable of handling infinite activity models, including unbounded variation processes. Exponential Lévy models are extensions of the classical Black and Scholes model in which the process of log-returns is generalized from an arithmetic Brownian motion to an arbitrary Lévy process (that is, a continuous-time stochastic process with independent stationary increments), thereby introducing jumps into the price process. Besides making prices behave more realistically, exponential Lévy models are capable of modelling the implied volatiltiy smile observed in the option markets, at least at fixed maturities. They fit certain empirical characteristics of financial time-series, notably excess kurtosis and non-Gaussian financial return distributions over both large and small time windows (the latter in contrast to a competing class of models capable of volatility smiles, the stochastic volatility models, which, as diffusion models, are approximately Gaussian over small time intervals). On the other hand, they retain the hypothesis of independent returns and do not address the issue of heteroscedasticity.

The introduction of exponential Lévy processes in option price modeling goes back to Merton's jump-diffusion model (Merton, 1976), which added an independent compound Poisson process to the classical Black and Scholes model. In recent years, a number of infinite activity Lévy processes, with and without a Brownian motion component, have been proposed for modeling financial asset returns: see for example (Madan and Seneta, 1990; Madan and Milne, 1991; Eberlein, 2001; Boyarchenko and Levendorskii, 2002a; Carr et al., 2002) and also (Schoutens, 2003; Cont and Tankov, 2004) for text-book treatments. In an infinite activity Lévy model the Lévy measure has a non-integrable singularity at the origin. As a consequence,

Correspondence Address: Email: r.brummelhuis@bbk.ac.uk and t.l.chan@uel.ac.uk 
infinite activity Lévy models will have infinitely many jumps of small size. They include pure jump processes whose sample paths a.s. have unbounded variation, and whose numerical treatment by Finite Difference methods have posed a particular challenge.

Since, in contrast to the classical Black and Scholes case, explicit formulas are in general not available for Lévy models (a notable exception being (b. Madan et al., 1998)), the problem of numerically pricing options in such models has attracted a lot of attention. Efficient numerical methods are indispensable for practical applications, such as fast calibration to market prices. The numerical methods employed till now roughly fall into three categories: Monte Carlo simulation, Fourier- or characteristic function methods and Finite Difference (FD) and Finite Element (FE) schemes. Monte Carlo methods require fast numerical simulation of Lévy trajectories, which is in itself a non-trivial problem. The other methods start from the observation that option prices prior to exercise satisfy a certain partial integro-differential equation or PIDE, which generalizes the classical Black and Scholes equation. The PIDE is defined in terms of the characteristic triple of the underlying Lévy process under a given risk-neutral measure: see equation (11) below.

Fourier transform methods for European options were introduced in (Carr and Madan, 1999), and extended to American options and weakly path-dependent options such as barrier options in (Jackson et al., 2008), under the name of Fourier Space Time-stepping or FST method. They are based on the observation that the option pricing PIDE is of convolution type, and can therefore be explicitly solved after taking Fourier transforms. The solution is then obtained by computing the inverse Fourier transform, which is numerically implemented using the Fast Fourier Transform or FFT. The FST is a flexible method which in numerical experiments shows second order convergence in the number of nodes used for the FFT. For an efficient implementation it does however require the characteristic exponent of the Lévy process to be known explicitly. Although Jackson et al. (2008) observe that the characteristic exponent, for a given Lévy measure, is always available in the form of the Lévy - Khintchine formula, this is as an oscillatory integral which would have to be evaluated numerically if it cannot be computed explicitly, thereby creating a further source of numerical errors. There therefore remains an interest in alternative methods which ony require knowledge of the Lévy measure itself ${ }^{1}$. Of these alternative methods, Finite Difference schemes have in particular received a lot of attention: see e.g. (Andersen and Andreasen, 2000; Almendral, 2004; Almendral and Oosterlee, 2005; 2006; 2007a;b; Hirsa and Madan, 2004; Tankov and Voltchkova, 2009; Cont and Voltchkova, 2005; d'Halluin et al., 2004; 2005; Hirsa and Madan, 2004; Wang et al., 2007). Their implementation for infinite activity Lévy processes presents something of a challenge. In a FD scheme we discretise the operator, and we in particular have to discretise the integral in the pricing PIDE. This is fairly straightforward if the Lévy measure is integrable, corresponding to a process of finite intensity, but a priori problematic if it has a non-integrable singularity at 0 . In that case, the integral is mostly split into a local part, containing the singularity of the Lévy measure, and a non-local part, which can be handled by classical quadrature techniques, such as the trapezoidal rule. The discretisation of the local part is more delicate, and in the most general case requires a second order Taylor expansion of the unknown function. The contribution of the small (or smallest) jumps is sometimes approximated by an effective diffusion terms, cf. (Cont and Voltchkova, 2005; Wang et al., 2007), although this procedure was criticised in (Levendorskii, 2004) and (Kudryavtsev and Levendorskii, 2009), where it was argued that it can lead to sizeable numerical errors. Most of the cited FD schemes use implicit time-stepping for the local part and explicit time-stepping for the nonlocal term, with the exception of (Wang et al., 2007) who use a fully impicit scheme. As far as convergence is concerned, the best results are those of (Wang et al., 2007),

${ }^{1}$ One can for example imagine a situation in which one would want to do a nonparametric calibration of the Lévy measure, the latter being given in spline form, or indeed in RBF-form, with unknown coefficients. 
which achieves second order convergence for finite variation Lévy processes, and slower than second order but better than first order, for infinite variation ones. An alternatives to FD was proposed by (Almendral and Oosterlee, 2007a), who rewrite the PIDE as a sum of two weakly singular Volterra operators through an integration by parts, and use established (but quite involved) numerical techniques to handle the latter. Their method shows second order convergence in numerical experiments with finite variation Lévy processes from the CGMY class but, as it stands, does not apply to infinite variation processes.

Finite element (FE) methods provide a viable alternative to FD, but seem for the moment less popular with practioners; see however (Achdou and Pironneau, 2010) and also (Hilber et al., 2013) for a recent comprehensive textbook treatment with applications to computational finance. (Matache et al., 2005a) implement and analyse a FE scheme with a wavelet basis and prove convergence of order 1 in space and at most 1 in time for the American option pricing problem, including for Lévy processes of unbounded variation. See also (Matache et al., 2005b) for a further application of wavelets in this context.

This paper uses an alternative scheme based on RBF-interpolation, in which we approximate the unknown solution $u=u(x, t)$ of the pricing PIDE $\partial_{t} u=\mathcal{L}[u]$ (with the operator $\mathcal{L}$ defined in (11) below) by time-dependent linear combinations

$$
u(x, t) \simeq \sum_{j} \rho_{j}(t) \varphi\left(x-x_{j}\right) .
$$

Here $\left(x_{j}\right)_{j}$ is a given finite sequence of interpolation points, and $\varphi$ a given radial basis function or RBF. The coefficients $\rho_{j}(t)$ are determined by a system of ODE found by requiring that our approximate solution exactly solves the PIDE in the points $x_{j}$. The scheme is therefore a variant of the classical method of lines. It is clearly not unrelated to the FE approach, though there are important differences: it uses point evaluations instead of $L^{2}$-projections, and thus belongs to the class of collocation methods. More importantly perhaps, unlike FE, where one uses well-localized basis functions, the radial basis function $\varphi$ can have non-compact support, and will in many cases even be growing at infinity, as for instance in this paper. For reasons explained in section 3 below, we will be using a stationary interpolation approach, in which the RBF will depend on the spacing of the interpolation points, which in this paper will simply be chosen on a regular grid. One advantage of the RBF approach (which it shares with FE and other Galerkin-type methods) is that we do not have to discretise the operator, and only have to compute the action of $\mathcal{L}$ on the, explicitly given, basis function $\varphi$ : we are using the fact that the PIDE is translation invariant. The explicit form of the basis function $\varphi$ can then be exploited to deal with the singularity of the Lévy measure. This leads to a simpler scheme than the aforementioned FD ones. As will be seen below, the RBF scheme achieves second order convergence in numerical examples, including processes whose sample paths are a.s. of unbounded variation. Establishing this rigorously is an open problem, which is outside of the scope of the present paper.

RBF schemes of the type used in this paper have been applied to parabolic PDEs such as the classical Black and Scholes PDE in one and two dimensions, mostly for European options: cf. (e.g. Fausshauer et al., 2004a;b; Hon and Mao, 1999; Larsson et al., 2008; Pettersson et al., 2008). (Chan, 2010) and (Chan, 2011) extended these studies to jump-diffusion models, which are finite activity. The present paper contributes to this growing literature by showing that the RBF scheme can deal with singular Lévy measures, without needing to make major modifications to the scheme, and by testing it on an important class of examples, the Lévymodels of the CGMY-KoBol class. A further aim of this paper is to make RBF-methods better known to practitioners and to the computational finance community in general. To make the paper self-contained, we start with a brief review of option pricing in exponential Lévy processes in section 2, and with a review of basic RBF interpolation theory at the 
beginning of section 3 . Section 3 continues with the explanation of how to handle singular Lévy measures, and with the formal description of the RBF schemes for both European and American options. Section 4 presents the results of our numerical experiments, and section 5 concludes.

\section{Financial modelling with Lévy processes}

We briefly review the theory of option pricing in Lévy-models, partly to establish notations. A standard reference for this material is (Cont and Tankov, 2004). Other references for the general theory of Lévy processes are (Applebaum, 2004), (Bertoin, 1996) and (Sato, 1999). We limit ourselves to derivatives written on a single risky asset whose log-return we assume to be modelled by a one-dimensional Lévy process. As usual, we also assume the existence of a risk-free bond earning interest at a constant rate of $r$. For a general Lévy-process, the market consisting of the risky asset plus the risk-free bond will be incomplete (notable exceptions are when the Lévy-process is a Brownian motion - the classical Black and Scholes model - or if it is a Poisson process), and there will exists infinitely many equivalent martingale measures under which prices of derivative assets are equal to discounted expectations of future pay-offs. As is customary in the Lévy-pricing literature, we will assume that the market has already chosen one of the possible risk-neutral measures, and expectations $\mathbb{E}$ will always be taken with respect to this chosen measure. The price of the risky asset is therefore given by

$$
S_{t}=S_{0} e^{L_{t}}, \quad t \geq 0,
$$

where $L_{t}, t \geq 0$, is a Lévy process under the given risk-neutral measure. If the asset also pays out a continuous dividend at a constant rate of $q$, then risk-neutrality means that

$$
\mathbb{E}\left(S_{t}\right)=S_{0} e^{(r-q) t}
$$

Remark 1: Although it is customary to model dividends as a continuous cash-stream with a known dividend-rate, in reality dividends are paid discretely. Discrete dividends such that both the ex-dividend dates and the dividend amounts are known in advance can easily be included in the usual option pricing models by splitting up the time domain according to the dividend dates, and then solving the pricing problem between each pair of consecutive dividend dates, starting with the final period up to maturity, with boundary conditions which are determined by the requirement that (i) just after the dividend falls, the underlying stock price decreases by the dividend payed, and (ii) for European options, the option price does not change across a dividend date (an easy consequence of Absence of Arbitrage). For American options we might choose to exercise just before or after a dividend is payed. The assumption of known dividends is reasonable for short-lived options, but (Hull, 2012) observes that for options with a long time-to-maturity it may be more appropriate to use a model with continuously payed dividends, with a dividend rate which is estimated from historical data. With stochastic dividends the modelling becomes more subtle. According to (Korn and Rogers, 2005), naive models for the joint distribution of stock price and dividends may lead to inconsistencies. They propose instead to model the discrete dividend process directly, define the stock price as the (risk-neutral) expected value of all discounted future dividend payments, and from there on compute option prices as usual, as discounted risk-neutral expectations - cf. 2.2 below.

\subsection{Characteristic triplets}

The Lévy-process $\left(L_{t}\right)_{t \geq 0}$ is fully determined by its characteristic function which, according to the Lévy-Khinchine theorem, is of the form $\mathbb{E}\left(e^{i z L_{t}}\right)=e^{t \psi(z)}$, with characteristic exponent 
$\psi(z)$ given by

$$
\psi(z)=i \gamma z-\frac{1}{2} \sigma^{2} z^{2}+\int_{\mathbb{R}}\left(e^{i x z}-1-i x z \mathbf{1}_{\{|x| \leq 1\}}\right) \nu(d x) .
$$

Here, $\gamma$ and $\sigma$ are real constants with $\sigma \geq 0$ and $\nu$ is a positive measure on $\mathbb{R}$, called the Lévy measure, which satisfies the Lévy-condition $\int_{\mathbb{R}} \min \left(x^{2}, 1\right) \nu(d x)<\infty$. The probabilistic interpretation of $\nu$ is that $\nu(d x)$ gives the expected number of jumps with size between $x$ and $x+d x$, which the process makes between time 0 and 1 . The triplet $(\gamma, \sigma, \nu)$ is called the characteristic triplet, or Lévy - Khintchine triplet, of $\left(L_{t}\right)_{t \geq 0}$.

The diffusion constant $\sigma$ and the Lévy-measure $\nu(d x)$ are canonically associated to the process $\left(L_{t}\right)_{t \geq 0}$, but $\gamma$ will depend on the way in which the (potential) singularity of the Lévy-measure in $x=0$ is regularized. One can for example replace $\mathbf{1}_{\{|x| \leq 1\}}$ in (3) by some other bounded function $\chi(x)$ such that $\chi(0)=1+O(x)$ : this would change $\gamma$ by an additional term of $-\int_{\mathbb{R}} x\left(\mathbf{1}_{|x| \leq 1}-\chi\right) \nu(d x)$, which is well-defined because of the Lévy-condition on $\nu$. $\mathrm{A}$ classical choice for $\chi$ was $\chi(x)=\left(1+x^{2}\right)^{-1}$.

In this paper all Lévy processes will be such that $|x|$ is $\nu$-integrable on $\{|x|>1\}$ :

$$
\int_{|x| \geq 1}|x| \nu(d x)<\infty
$$

in which case we can rewrite (3) as

$$
\psi(z)=i \gamma_{c} z-\frac{1}{2} \sigma^{2} z^{2}+\int_{\mathbb{R}}\left(e^{i z x}-1-i z x\right) \nu(d x),
$$

with $\gamma_{c}=\gamma+\int_{|x|>1} x \nu(d x)$. In case the Lévy measure satisfies the stronger integrability condition

$$
\int_{|x| \leq 1}|x| \nu(d x)<\infty
$$

the Lévy-Khintchine formula can be further simplified to

$$
\psi(z)=i \beta z-\frac{1}{2} \sigma^{2} z^{2}+\int_{\mathbb{R}}\left(e^{i z x}-1\right) \nu(d x),
$$

where

$$
\beta=\gamma_{c}-\int_{\mathbb{R}} x \nu(d x) .
$$

Condition (6) is equivalent to the process' sample paths being a.s of finite variation; cf. (Sato, 1999, Theorem 21.9.).

It is known that $S_{t}=e^{L_{t}}$ is integrable (has finite expectation) for all $t>0$ iff this is the case for $t=1$, and that this is equivalent to $\int_{|x| \geq 1} e^{x} \nu(d x)<\infty$ (cf. Cont and Tankov, 2004, Proposition. 3.14) or (Sato, 1999, Theorem 25.17). In that case (remembering (4)), $\psi$ will extend to a holomorphic function on the strip $\{-1 \leq \operatorname{Im} z \leq 0\}$ and $\mathbb{E}\left(S_{t}\right)=\exp (t \psi(-i))$. It follows that the risk-neutrality condition (2) is equivalent to

$$
\psi(-i)=\gamma_{c}+\frac{1}{2} \sigma^{2}+\int_{\mathbb{R}}\left(e^{x}-1-x\right) \nu(d x)=r-q .
$$

This fixes $\gamma_{c}$ for given $\sigma$ and $\nu(d x)$. To specify a risk-neutral model it therefore suffices to specify the latter two. This paper mostly considers pure jump processes, for which $\sigma=0$. 


\subsection{European option pricing}

By risk-neutral pricing, the price at time $t$ of a European option paying out $G\left(S_{T}\right)$ at maturity $T \geq t$, given that $S_{t}=S$, is equal to

$$
e^{-r \tau} \mathbb{E}\left(G\left(S_{T}\right) \mid S_{t}=S\right)=e^{-r \tau} \mathbb{E}\left(G\left(S e^{L_{\tau}}\right)\right),
$$

where $\tau:=T-t$ is the time-to-maturity, and where we used stationarity and independence of increments of $L_{t}$. Writing the option price as a function of the $\log$-price $x:=\log S$ and of $\tau$,

$$
u(x, \tau):=e^{-r \tau} \mathbb{E}\left(G\left(e^{x+L_{\tau}}\right)\right),
$$

it can be shown that $u$ satisfies the following Partial Integro-Differential Equation or PIDE

$$
\begin{aligned}
\partial_{\tau} u(x, \tau) & =\frac{1}{2} \sigma^{2} \partial_{x}^{2} u+\gamma_{c} \partial_{x} u-r u+\int_{\mathbb{R}}\left(u(x+y, \tau)-u(x, \tau)-y \partial_{x} u(x, \tau)\right) \nu(d y) \\
& =: \mathcal{L}[u](x, \tau),
\end{aligned}
$$

with initial value $u(x, 0)=G\left(e^{x}\right)$; cf. (Cont and Tankov, 2004), for a proof based on Ito's formula for general semi-martingales, and also (Revuz and Yor, 1999), proposition (1.9) in Chapter VII, for an elementary proof using only the Lévy-Khinchine formula and the tempered Fourier transform (observe that the right hand side of (11) commutes with translations). For a European call or put option, the appropriate initial conditions are $u(x, 0)=\max \left(e^{x}-K, 0\right)$ respectively $u(x, 0)=\max \left(K-e^{x}, 0\right)$.

Remark 2: Using the risk-neutrality condition (9), the operator $\mathcal{L}$ can be re-written as

$$
\frac{1}{2} \sigma^{2} \partial_{x}^{2} u+\left(r-q-\frac{1}{2} \sigma^{2}\right) \partial_{x} u-r u+\int_{\mathbb{R}}\left(u(x+y, \tau)-u(x, \tau)-\left(e^{y}-1\right) \partial_{x} u(x, \tau)\right) \nu(d y),
$$

which is the form in which the PIDE is derived in (Cont and Tankov, 2004).

Remark 3: If the Lévy process has sample paths which are a.s. of finite-variation, then, using $(7), \mathcal{L}[u]$ can be given the simpler expression

$$
\mathcal{L}[u](x, \tau)-\frac{1}{2} \sigma^{2} \partial_{x}^{2} u+\beta \partial_{x} u-r u+\int_{\mathbb{R}}(u(x+y, \tau)-u(x, \tau)) \nu(d y),
$$

and the risk-neutrality condition (9) becomes $\beta+\frac{1}{2} \sigma^{2}+\int\left(e^{x}-1\right) \nu(d x)=r-q$. This situation covers important Lévy-models such as the Variance-Gamma model, or the CGMY-KoBoL model with $Y<1$.

Remark 4: A minor technical point is that solutions to parabolic Cauchy-problems such as (11) need not be unique, unless additional growth conditions in $x$ are imposed on the solution. In Finance, natural growth conditions are provided by the principle of Absence of Arbitrage. For example, for a put the latter implies that $0 \leq u(x, \tau) \leq K$, and bounded solutions of the Cauchy problem (11) with bounded initial value will be unique, as can easily be shown using the (tempered) Fourier transform. Similarly, for a call, uniqueness is assured by $0 \leq u(x, \tau) \leq e^{x}$ (as can be shown using the complex Fourier transform, assuming, as we do, that $e^{x}$ and $|x|$ are $\nu$-integrable on $\{|x| \geq 1\}$ ), which again is implied by Absence of Arbitrage.

\subsection{Pricing American options}

American options distinguish themselves from European ones in that the holder can choose to exercise the option at any time prior to maturity. We will limit our discussion to American 
put options. The arbitrage-free price of the American put at time 0 is given by

$$
\begin{aligned}
V^{\mathrm{A}}(S, 0) & =\sup _{0 \leq \mathfrak{T} \leq T} \mathbb{E}\left(e^{-r \mathfrak{T}} \max \left(K-S_{\mathfrak{T}}, 0\right) \mid S_{t}=S\right) \\
& =\sup _{0 \leq \mathfrak{T} \leq T} \mathbb{E}\left(e^{-r \mathfrak{T}} \max \left(K-S e^{L_{\mathfrak{T}}}, 0\right)\right),
\end{aligned}
$$

$\mathfrak{T}$ ranging over the stopping times of the Lévy-process $L$ (stopping times are often denoted by $\tau$, but we already used $\tau$ for time-to-maturity, whence the Gothic $\mathfrak{T}$ ).

The American put problem can be reformulated as a variational inequality or as a free boundary problem. For the latter, care needs to be taken with the pasting condition on the free boundary: smooth pasting is not always valid for pure jump processes, and sometimes needs to be weakened to continuous pasting only: see (Boyarchenko and Levendorskii, 2002b; Alili and Kyprianou, 2005) and also (Peškir and Shiryaev, 2006). We will examine this point numerically in section 4 below. In this paper we will use neither of these reformulations, but proceed directly from (13) by discretising time and numerically solving the resulting discrete time optimal stopping problem using backward induction. Specifically, we take a sufficiently fine partition $\mathcal{P}:=\left\{t_{0}=0<t_{1}<\cdots<t_{M}=T\right\}$ of $[0, T]$ and determine the discrete-time value-functions

$$
V_{\mathcal{P}}^{\mathrm{A}}\left(S, t_{m}\right):=\sup _{\mathfrak{T} \in\left\{t_{m}, \ldots, t_{M}\right\}} \mathbb{E}\left(e^{-r \mathfrak{T}} \max \left(K-S e^{L_{\mathfrak{T}}}, 0\right)\right)
$$

using the familiar backwards induction procedure:

$$
V_{\mathcal{P}}^{\mathrm{A}}\left(S, t_{m}\right)=\max \left(e^{-r\left(t_{m+1}-t_{m}\right)} \mathbb{E}\left(V_{\mathcal{P}}^{\mathrm{A}}\left(S e^{L_{t_{m+1}}-L_{t_{m}}}, t_{m+1}\right)\right), \max (K-S, 0)\right),
$$

starting off with $V_{\mathcal{P}}^{\mathrm{A}}(S, T)=\max (K-S, 0)$. It is known (cf. (Zhang, 1997, lemma 3.5) or (Peškir and Shiryaev, 2006)) that $V_{\mathcal{P}}^{\mathrm{A}}$ will converge to $V^{\mathrm{A}}$ as the partition's width $\max _{m}\left(t_{m+1}-t_{m}\right) \rightarrow 0$. We will compute the expectation on the right hand side by (numerically) solving the pricing PIDE on $\left[t_{m}, t_{m+1}\right]$ with final pay-off $V^{\mathrm{A}}\left(S, t_{m+1}\right)$. Transforming to log-price and time-to-maturity coordinates $(x, \tau)$ we arrive at the following scheme for $u_{\mathcal{P}}^{\mathrm{A}}\left(x, \tau_{m}\right):=V_{\mathcal{P}}^{\mathrm{A}}\left(e^{x}, T-\tau_{m}\right)$, the (approximate) put-value as function of log-price at time-tomaturity $\tau_{m}:=T-t_{m}$ :

(i) $u_{\mathcal{P}}^{\mathrm{A}}(x, 0)=\max \left(K-e^{x}, 0\right)$

(ii) For $m=1, \ldots, M, u_{\mathcal{P}}^{\mathrm{A}}\left(x, \tau_{m}\right)=\max \left(u_{m}\left(x, \tau_{m}\right), K-e^{x}\right)$, where $u_{m}(x, \tau)$ solves

$$
\left\{\begin{array}{l}
\partial_{\tau} u_{m}(x, \tau)=\mathcal{L}\left[u_{m}\right](x, \tau), \tau \geq \tau_{m-1} \\
u_{m}\left(x, \tau_{m-1}\right)=u_{\mathcal{P}}^{\mathrm{A}}\left(x, \tau_{m-1}\right)
\end{array}\right.
$$

The American put value at time 0 will then be approximated by $V_{\mathcal{P}}^{\mathrm{A}}(S, 0)=u_{\mathcal{P}}^{\mathrm{A}}\left(\log S, t_{M_{0}}\right)=$ $u_{\mathcal{P}}^{\mathrm{A}}(x, T)$. In section 4 , we will numerically solve the sequence of Cauchy problems (16) using an RBF scheme.

\subsection{The CGMY and VG processes}

We will test our RBF schemes on Lévy processes from the well-known CGMY-KoBoL class (Koponen, 1995; Boyarchenko and Levendorskii, 2002a; Carr et al., 2002) . These are pure jump processes $(\sigma=0)$ with Lévy measure given, in the notation of (Carr et al., 2002), by

$$
\nu_{\mathrm{CGMY}}(d x):=\frac{k_{\mathrm{CGM}}(x)}{|x|^{Y+1}} d x
$$


with

$$
k_{C G M}(x):=\left\{\begin{array}{l}
C \exp (-G|x|), x<0, \\
C \exp (-M x), x>0,
\end{array}\right.
$$

where $C, G, M>0$ and $Y<2$ (KoBoL allows the constant $C$ to differ for $x>0$ and $x<0$.). The special case of $Y=0$ defines the subclass of Variance-Gamma or VG processes, which were introduced earlier by (Madan and Seneta, 1990) as a time-changed Brownian motion. The VG process was originally defines as an arithmetic Brownian motion $\theta t+\sigma W_{t}$ evaluated in a random time given by an independent Gamma process $\Gamma_{t}(1, v$ of mean rate 1 and variance rate $v$, and adding a drift-term $\beta t: L_{t}^{\mathrm{VG}}:=\beta t+\theta \Gamma_{t}(1, v)+\sigma W_{\Gamma_{t}}$. The characteristic exponent of the VG-process is known to be $i \beta z-\frac{1}{v} \log \left(1-i \theta v z+\frac{1}{2} \sigma^{2} v z^{2}\right)$, and its CGMY-parameters are

$$
C=v^{-1}, G^{-1}=\frac{1}{2}\left(\sqrt{\theta^{2} v^{2}+2 \sigma^{2} v}-\theta v\right), M^{-1}=\frac{1}{2}\left(\sqrt{\theta^{2} v^{2}+2 \sigma^{2} v}+\theta v\right), Y=0 .
$$

Since $Y=0$, the Lévy measure of a VG process satisfies (6), and we can use the simpler form (12) for $\mathcal{L}[u]$ (with $\sigma=0$ ). The risk-neutrality condition $\psi(-i)=r-q$ translates into

$$
\beta=r-q+\frac{1}{v} \log \left(1-\theta v-\frac{1}{2} \sigma^{2} v\right) .
$$

Amongst numerous other references, VG processes were further studied in (Madan and Milne, 1991; Carr et al., 1998) .

Returning to general CGMY processes, their characteristic functions can be explicitly computed (see for example (Cont and Tankov, 2004), Chapter 4.5). As a consequence, the riskneutral drift $\gamma_{c}$ can be expressed in terms of the CGMY-parameters and of $r$ and $q$, using (9). We state the result for completeness:

$$
\gamma_{c}=\left\{\begin{array}{l}
r-q+C\left(\log \left(\left(1+G^{-1}\right)\left(1-M^{-1}\right)\right)-G^{-1}+M^{-1}\right), \quad Y=0, \\
r-q-C\left((1+G) \log \left(1+G^{-1}\right)+(M-1) \log \left(1-M^{-1}\right)\right), \quad Y=1 \\
r-q-C \Gamma(-Y)\left(G^{Y}\left(\left(1+G^{-1}\right)^{Y}-1-G^{-1} Y\right)+M^{Y}\left(\left(1-M^{-1}\right)^{Y}-1+M^{-1} Y\right)\right),
\end{array}\right.
$$

where $Y \in(0,2) \backslash\{1\}$ for the latter, and where we asumed $M>1$.

The parameter $Y$ determines the nature of the paths of the CGMY-process: these are a.s. of bounded variation iff $Y<1$, and the process has finite activity (that is, its paths a.s. only have a finite number of jumps in any finite time interval) iff $Y<0$. If $Y<1, \mathcal{L}[u]$ can be converted to the simplified form (12), with

$$
\beta=\gamma_{c}-\int_{\mathbb{R}} x \nu_{\mathrm{CGMY}}(d x)=\gamma_{c}+C \Gamma(1-Y)\left(G^{Y-1}-M^{Y-1}\right) .
$$

For some of the numerical experiments of section 4 below we used a trivially extended CGMY-model, obtained by adding an independent Brownian motion $\sigma W_{t}$ (not to be confused with the Brownian motion underlying the VG process).

\section{An RBF Algorithm for Pricing American and European Options}

\subsection{Radial Basis Function or RBF interpolation}

We next briefly review basic RBF interpolation theory. General references for this subsection are the books (Buhmann, 2003) and (Wendland, 2005). Radial basis function-, or RBF - 
interpolation seeks to interpolate arbitrary real- (or complex-) valued functions $f$ on $\mathbb{R}^{n}$ by linear combinations of translates of some given basis function $\varphi$. Specifically, for a given set of interpolation points $X=\left\{x_{1}, \ldots, x_{N}\right\}$ one tries to find constants $\alpha_{1}, \ldots, \alpha_{N}$ such that $s_{X}[f](x):=\sum_{j=1}^{N} \alpha_{j} \varphi\left(x-x_{j}\right)$ satisfies:

$$
s_{X}[f]\left(x_{i}\right)=f\left(x_{i}\right), \quad i=1, \ldots, N .
$$

Regarded as an $N \times N$ system of linear equations in the $\alpha_{j}$ 's, (20) will have a unique solution $\alpha=\left(\alpha_{1}, \ldots, \alpha_{N}\right)$ iff the $N \times N$ coefficient matrix

$$
\mathbf{\Phi}_{X}:=\left(\varphi\left(x_{i}-x_{j}\right)\right)_{1 \leq i, j \leq N},
$$

is non-singular. A natural condition under which this is true for any choice of interpolation points $x_{i}$ is that $\varphi$ be positive definite. If $\varphi$ is integrable this is equivalent, by Bochner's theorem, to its Fourier transform being positive. The basis function $\varphi$ is generally assumed to be radial: this is often not strictly speaking necessary, but is numerically advantageous, especially in higher dimensions, since it simplifies the numerical evaluation of $\varphi\left(x_{i}-x_{j}\right)$. If we write $\varphi(x)=\phi\left(|x|^{2}\right)$, where $|\cdot|$ is the Euclidean norm, then $\varphi$ will be positive definite if $\phi: \mathbb{R}_{>0} \rightarrow \mathbb{R}$ is the Laplace transform of a positive finite Borel measure on $\mathbb{R}_{>0}$; the converse of this statement is also true: cf. (Buhmann, 2003). Examples of RBFs obtained in this way are the Gaussians and the generalized inverse multi-quadrics $\left(c+|x|^{2}\right)^{-\alpha}(\alpha>0)$, which correspond to $\phi(r)=(c+r)^{-\alpha}=\int_{0}^{\infty} e^{-s r} g(s) d s$, with $g(s)=\Gamma(\alpha)^{-1} s^{\alpha-1} e^{-c s}$. The inverse multi-quadric corresponding to $\alpha=1 / 2$ was used in the earlier study (Chan, 2010) of Merton's jump-diffusion model using RBF-techniques. RBF-interpolation is an efficient and easily-to-implement general technique for interpolation and approximation, which can serve as a flexible alternative to classical interpolation techniques such as polynomial interpolation or splines, especially in higher dimensions, where more traditional methods either fail or become more difficult to implement. Its main potential drawback lies in the condition number of the coefficient matrix $\boldsymbol{\Phi}_{X}$ becoming high when $X$ becomes increasingly dense, making the system (20) ill-conditioned.

One often uses non-integrable basis functions $\varphi$ such as the generalised multiquadric $(1+$ $\left.|x|^{2}\right)^{\kappa}$ with $\kappa \notin \mathbb{N}$ (if $\kappa$ is a positive integer, this reduces to a polynomial). In fact, the use of such non-integrable RBFs practically imposes itself when performing so-called stationary interpolation, in which the basis function $\varphi$ is adapted to the scale of the interpolation set $X$, as measured for example by the minimum distance between its points. A typical example of stationary interpolation is interpolation on a regular grid $X=h \mathbb{Z} \subset \mathbb{R}$ with spacing $h$, using a basis function of the form $\varphi_{h}(x):=\varphi_{1}(x / h)$ with $\varphi_{1}$ fixed. This choice of basis function has the advantage of making the coefficient matrix $\boldsymbol{\Phi}_{X}$ of the system (20) independent of the scale $h$, which adresses conditioning problems which can otherwise occur for small $h$. Using integrable $\varphi_{1}$ would however lead to bad convergence properties of $s_{X}[f]$ to $f$ when $h \rightarrow 0$ : to have convergence, one basically needs the generalised Fourier transform of $\varphi_{1}$ to be singular in 0: cf. (Buhmann, 2003), Chapter 4, for further explanations. For interpolation with a non-integrable RBF, the notion of positive (or negative) definiteness is no longer the appropriate one, and has to be replaced by that of conditionally positive (or negative) definiteness: $\varphi$ is called conditionally positive (or negative) definite of degree $k$ if for any subset $X=\left\{x_{1}, \ldots, x_{N}\right\} \subset \mathbb{R}^{n}$, the matrix $\boldsymbol{\Phi}_{X}$ is positive (or negative) definite on the subspace of all $\alpha=\left(\alpha_{1}, \cdots, \alpha_{N}\right) \in \mathbb{R}^{N}$ such that $\sum \alpha_{j} p\left(x_{j}\right)=0$ for all polynomials $p(x)$ of degree $k-1$. One then in general has to add a polynomial of order $k-1$ to the interpolating function $s_{X}[f]$, and add further constraints on the $x_{j}$ to keep unique solvability: we refer to Appendix A for details.

Since our RBF algorithm for solving the pricing PIDE will use stationary interpolation, and good convergence properties of the basic RBF-interpolation algorithm will be important 
for the convergence of the scheme (if only since the scheme will start off by determining an RBF-interpolant of the initial value), we need to use a non-integrable basis function, for which we have chosen the multi-quadric,

$$
\varphi(x):=\varphi_{c}(x):=\sqrt{|x|^{2}+c^{2}},
$$

where $c>0$. The multi-quadric is known to be conditionally negative definite of order 1 , and $\boldsymbol{\Phi}_{X}$ will therefore be negative definite on the orthogonal complement of the vector $(1, \ldots, 1)$, which is a subspace of codimension 1 . Since the trace of $\boldsymbol{\Phi}_{X}$ is strictly positive (it equals $N c$ ), the remaining eigenvalue of $\boldsymbol{\Phi}_{X}$ has to be strictly positive, so that $\boldsymbol{\Phi}_{X}$ will be invertible, and the system (20) will still be uniquely solvable, without having to augment $s_{X}[f]$ by a 0 -th order polynomial or constant term.

Stationary RBF-interpolation with a multi-quadric on a regular grid has good approximation properties: one can show that $\left\|s_{h}[f]-f\right\|_{\infty} \leq C h^{2}$ if $f \in C^{1}(\mathbb{R})$ such that $f^{\prime}$ is Lipschitz, as follows by combining theorems 4.2, 4.3 and 4.5 of (Buhmann, 2003). The multi-quadric provides a good compromise between having good stationary approximation and keeping the basic RBF-algorithm simple, on account of the invertibility of the $\boldsymbol{\Phi}_{X}$ 's.

The constant $c$ is called the shape parameter of the multi-quadric: it strongly influences the condition number of the linear system (20). There exists an extensive literature on finding the optimal shape parameter $c$ for multiquadrics and other RBFs: see for example (Fasshauer and Zhang, 2007; Fornberg and Wright, 2004; Kansa and Carlson, 1992).

Non-stationary interpolation schemes, in which one fixes the $\operatorname{RBF} \varphi$ while letting the set of interpolation points become dense, can have extremely good approximation properties. Depending on the function $f$, one can even achieve exponential rates of convergence rates on compact subsets of $\mathbb{R}^{n}$, for example if $f$ belongs to the so-called native space of $\varphi$, meaning that $\widehat{f} / \sqrt{\widehat{\varphi}}$ is square-integrable (the hat indicating Fourier transform): see Madych (1992); Buhmann and Dyn (1993). However, the lowest eigenvalue of the coefficient matrix $\boldsymbol{\Phi}_{X}$ of the system (20) can become exponentially small with increasingly dense interpolation sets $X$, leading to large condition numbers: see for example (cf Fasshauer, 2007, chapter 16, example 16.2). The so-called uncertainty principle of Shaback, cf. (Schaback, 1994; 1995), shows there is a reciprocal trade-off between condition number and sup-norm approximation error: they cannot both be small. We note that Shaback's uncertainty principle is valid for the standard formulation of RBF interpolation. There are reformulations which are less sensitive with respect to conditioning: see for example (Fornberg et al., 2011) and (Fasshauer and McCourt, 2012).

Non-stationary RBF algorithms for classical Black and Scholes PDE (in one and two dimensions) have been studied in (Pettersson et al., 2008). In this paper we have limited ourselves to stationary interpolation.

\subsection{An RBF-scheme for Cauchy problems}

For a given radial basis function $\varphi(x)$ and a given set of interpolation, or collocation, points $X=\left\{x_{1}, \ldots, x_{N}\right\} \subset \mathbb{R}$, we look for an approximate solution to our Cauchy-problem

$$
\left\{\begin{array}{l}
\partial_{\tau} u=\mathcal{L}[u], \quad \tau>0 \\
u(x, 0)=g(x)
\end{array}\right.
$$

of the form

$$
U_{X}(x, \tau):=\sum_{j=1}^{N} \rho_{j}(\tau) \varphi\left(x-x_{j}\right)
$$


whee $\mathcal{L}$ is given by (11). To determine the vector of coefficients $\boldsymbol{\rho}(\tau):=\left(\rho_{1}(\tau), \ldots, \rho_{N}(\tau)\right)$ we use a variant of the method of lines, and derive a system of ODEs for $\rho(\tau)$ by requiring that

$$
\partial_{\tau} U_{X}\left(x_{i}, \tau\right)=\mathcal{L}\left[U_{X}\right]\left(x_{i}, \tau\right) \quad 0 \leq i \leq N, \tau \geq 0 .
$$

Since $\mathcal{L}$ commutes with translations, we find that

$$
\mathcal{L}\left[U_{X}\right](x, \tau)=\sum_{j=1}^{N} \rho_{j}(\tau) \mathcal{L}[\varphi]\left(x-x_{j}\right) .
$$

and since $\partial_{\tau} U_{X}(x, \tau)=\sum_{j}\left(d \rho_{j} / d \tau\right) \varphi\left(x-x_{j}\right),(25)$ is equivalent to

$$
\boldsymbol{\Phi}_{X} \frac{d}{d \tau} \boldsymbol{\rho}(\tau)=(\mathbf{L} \boldsymbol{\Phi})_{X} \boldsymbol{\rho}(\tau)
$$

where $\boldsymbol{\Phi}_{X}$ was defined in (21) above, and where the $N \times N$ matrix $(\mathbf{L} \boldsymbol{\Phi})_{X}$ is defined by

$$
(\mathbf{L} \boldsymbol{\Phi})_{X}:=\left(\mathcal{L}[\varphi]\left(x_{i}-x_{j}\right)\right)_{1 \leq i, j \leq N} .
$$

Assuming that our RBF $\varphi$ is such that $\boldsymbol{\Phi}_{X}$ is invertible, we find that $\boldsymbol{\rho}(\tau)$ has to satisfy the following system of ODEs:

$$
\frac{d}{d \tau} \boldsymbol{\rho}(\tau)=\boldsymbol{\Phi}_{X}^{-1}(\mathbf{L} \boldsymbol{\Phi})_{X} \boldsymbol{\rho}(\tau)
$$

It is natural to require that $\sum_{j} \rho_{j}(0) \varphi\left(x-x_{j}\right)$ is the RBF interpolant of initial value function $g(x)$, which leads to the initial condition

$$
\boldsymbol{\rho}(0)=\left.\boldsymbol{\Phi}_{X}^{-1} g\right|_{X},
$$

where $\left.g\right|_{X}:=\left(g\left(x_{1}\right), \ldots, g\left(x_{N}\right)\right)$. The initial value problem (29), (30) for $\boldsymbol{\rho}$ can be solved numerically using an appropriate ODE solver. The system (29) is a simple linear constant coefficient system of ODEs. Its numerical solution requires however some care, since it generally will be a stiff system: the coefficient matrix can have a large stiffness ratio, defined as the quotient of its largest and smallest, in absolute value, eigenvalues. For example, the stiffness ration of $\boldsymbol{\Phi}_{X}^{-1}(\mathbf{L} \boldsymbol{\Phi})_{X}$ computed on a set of 200 equally spaced points in the interval $[-9,9]$ for a multiquadric with $c=4 h$ ( $h$ being the spacing between the points) was found to be of the order of $2 \cdot 10^{9}$. For such systems a given numerical method can require extremely small step-sizes to avoid instability, much smaller than one would normally expect to have to use to attain a given precision: see for example (Iserles, 2009) for further discussion. Stiff systems generally have to be solved by an implicit method. We used custom-made matlab software which implements a backward differentiation (BDF) scheme of order 2 appropriate for stiff ODEs. We did not explicitly investigate the errors coming from the time-discretisation of the ODE system (29), since the BDF scheme can in principle handle this to any desired precision, but have concentrated on the discretisation error in the $x$-variable, since we foremost wanted to investigate the performance of the RBF-leg of the algorithm.

Remark 1: Note that our algorithm applies to any translation-invariant linear operator $\mathcal{L}$ having $\varphi$ in its domain, and that we only need to compute the action of $\mathcal{L}$ on $\varphi$. Also note that the RBF-scheme does not discretise the operator, in contrast to Finite Difference schemes (but in common with for example Galerkin-type schemes). This will considerably simplify the numerical treatment of singularities which may be present in the integral kernel of the operator $\mathcal{L}$ (as for example with infinity activity Lévy models).

Remark 2: In the more general case of a general conditionally (positive) definite radial basis functions, $\boldsymbol{\Phi}_{X}$ will only be positive definite on a subspace of codimension 1 or more, and our RBF-ansatz (24), as well as the basic algorithm (29), (30), will have to be modified accordingly: this is worked out in Appendix A. 


\subsection{Singularity of the Lévy measure}

The various Finite Difference (FD) schemes which have been proposed in the literature to solve the PIDE (11) all have to address the problem of how to discretise the operator $\mathcal{L}$ in a neigborhood of the singularity of the Lévy measure, when the Lévy-process is infinite activity. This is commonly dealt with by splitting the integral into a local part near the singularity, and a non-local part, and using a Taylor expansion of the unknown function $u$ near the singularity. The contribution of small jumps $|y| \leq \varepsilon$ is sometimes approximated by an effective diffusion term $\sigma(\varepsilon)^{2} \partial_{y}^{2}$. The non-local part is computed using a numerical quadrature scheme, sometimes using FFT to speed up the computations (note that the integral is of convolution type, making it amenable to Fourier analysis). See for example (Hirsa and Madan, 2004), (Almendral and Oosterlee, 2007b), (Almendral, 2004), (Cont and Voltchkova, 2005), (Wang et al., 2007). An alternative approach is that of (Almendral and Oosterlee, 2007a), where the integral operator was rewritten as a sum of two weakly singular Volterra operators acting on $\partial_{x} u$.

An advantage of the RBF method is that we only have to compute the action of the integral operator on a single function, the radial basis function $\varphi$. Since $\varphi$ is explicit (in our case, a multi-quadric) we can exploit its properties to explicitly de-singularise the integral, and convert it to a form which is amenable to classical quadrature techniques.

Lemma 3.1: $\operatorname{Let} \varphi(x):=\varphi_{c}(x)=\sqrt{x^{2}+c^{2}}$. Then

(i) $\varphi(x+y)-\varphi(y)=y f_{\mathrm{MQ}}^{(1)}(x, y)$, where

$$
f_{\mathrm{MQ}}^{(1)}(x, y)=\frac{y+2 x}{\varphi(x+y)+\varphi(x)}
$$

(ii) $\varphi(x+y)-\varphi(x)-y \varphi^{\prime}(x)=y^{2} f_{\mathrm{MQ}}^{(2)}(x, y)$, where

$$
f_{\mathrm{MQ}}^{(2)}(x, y)=\frac{1}{\varphi(x+y)+\varphi(x)}-\frac{2 x^{2}+x y}{\varphi(x)(\varphi(x+y)+\varphi(x))^{2}} .
$$

Proof. Straightforward computation.

The point of this lemma is that the powers of $y$ on the right will cancel out the singularity in $y=0$ of $\nu(d y)$ in (11). One can use (ii) for a general Lévy measure, and (i) if $\nu$ satisfies (4); cf. (12). Specifically, for the CGMY-model we get

$$
\mathcal{L}[\varphi](x)=\gamma_{c} \partial_{c} \varphi-r \varphi+\int_{\mathbb{R}} f_{\mathrm{MQ}}^{(2)}(x, y) k_{\mathrm{CGM}}(y)|y|^{1-Y} d y
$$

Note that if $Y \leq 1$, we have a continuous integrand. Alternatively, if $Y \leq 1$, we could also have used (31) together with (12) to write

$$
\int_{\mathbb{R}} f_{\mathrm{MQ}}^{(1)}(x, y) k_{\mathrm{CGM}}(y) \frac{\operatorname{sgn}(y)}{|y|^{Y}} d y
$$

for the integral part of $\mathcal{L}[\varphi](x)$, which has an integrable singularity at 0 . Numerically this singularity would however need special treatment, while (33) can be dealt with by classical quadrature methods (note that the integrand is in fact smooth on both the positive and negative closed half-line, though not in 0 ). If $1<Y<2$ we again encounter an integrable singularity in (33), which we can remove by a simple integration by parts, separately on the 
two intervals $\mathbb{R}_{>0}$ and on $\mathbb{R}_{<0}$. For example,

$$
\begin{aligned}
& \int_{0}^{\infty} y^{1-Y} f_{\mathrm{MQ}}^{(2)}(x, y) k_{\mathrm{CGM}} d y \\
= & -\int_{0}^{\infty} \frac{y^{2-Y}}{2-Y} \partial_{y}\left[k_{\mathrm{CGM}}(y) f_{\mathrm{MQ}}^{(2)}(x, y)\right] d y .
\end{aligned}
$$

where the boundary terms vanish since $2-Y>0$ and since $k_{\mathrm{CGM}}$ is exponentially decreasing at infinity. The remaining integral has a (left-) continuous (and even infinitely differentiable) integrand on $\{y \geq 0\}$ which decays exponentially at $\pm \infty$ and which can be handled by classical quadrature methods. We have used adaptive Gauss-Kronrod quadrature (Shampine, 2008). Given that $k_{\mathrm{CGM}}$ is a (constant times a) negative exponential, Gauss-Laguerre quadrature would have been a natural alternative.

Remark 3: If the Lévy measure is explicitly known, as it is for CGMY, one might try to go one step further, and compute $\mathcal{L}[\varphi]$ in terms of special functions. We did not succeed in doing this here. Even if such a computation can be carried out, the question arises of whether the special functions in question can be efficiently evaluated, which is not always trivial.

With all elements in place, we can now more formally present our RBF-scheme for European and American options in an exponential Lévy model.

\subsection{RBF-algorithm for Europan options}

1. Choose a computational range $\left[x_{\min }, x_{\max }\right]$ in log-price space and interpolation points $X=\left\{x_{1}, \ldots, x_{N}\right\} \subset\left[x_{\min }, x_{\max }\right]$.

2. Choose an $\operatorname{RBF} \varphi$ (possibly depending on $X$ ) and compute the matrices $\boldsymbol{\Phi}_{X}$ and $(\mathbf{L} \boldsymbol{\Phi})_{X}$ given by (21) and (28), using lemma 3.1 if the Lévy measure is singular in 0 .

3. Determine the RBF-interpolant $\sum_{j} \rho_{j}(0) \varphi\left(x-x_{j}\right)$ of the initial value $g(x)=G\left(e^{x}\right)$, where $G$ is the payoff function.

4. Solve the linear constant coefficient system of ODEs (29) with initial vector $\boldsymbol{\rho}(0)=$ $\left(\rho_{1}(0), \ldots, \rho_{N}(0)\right)$ up to time $T$, using an ODE-solver for stiff ODEs.

5. Put $U(x, T):=U_{X}(x, T):=\sum_{j} \rho_{j}(T) \varphi\left(x-x_{j}\right)$.

$U_{X}(x, T)$ is then the RBF-approximation of the time- 0 option price using the set of interpolation points $X$ (and the RBF function $\varphi$, which we suppress from the notation).

Similar algorithms have been studied for one- and multi-dimensional Black and Scholes models (e.g. Fausshauer et al., 2004a;b; Hon and Mao, 1999; Larsson et al., 2008; Pettersson et al., 2008) In these cases, $\mathcal{L}$ is a second order elliptic differential operator, and the computation of $(\mathbf{L} \boldsymbol{\Phi})_{X}$ is straightforward. For jump-diffusions, which are finite activity, this algorithm (and its companion version for American options below) has been implemented and studied in (Chan, 2010; 2011) with different choices of basis functions (inverse multi-quadric and cubic spline, respectively).

Remark 4: Note that we assumed that $\boldsymbol{\Phi}_{X}$ is invertible. If $\varphi$ is conditionally positive definite with $\boldsymbol{\Phi}_{X}$ invertible only on a subspace of positive co-dimension, the algorithm has to be modified along the lines of Appendix A.

For $X$ we will simply take a set of equidistant points $X=X_{N}:=\left\{x_{1}^{h}, \ldots, x_{N}^{h}\right\}$ with

$$
x_{j}^{h}:=x_{\min }+j h, \quad j=0, \ldots N,
$$

where $h=\left(x_{\max }-x_{\max }\right) / N$. Other choices are of course possible: (Pettersson et al., 2008) work with a non-uniform set of points which are more densely (though still uniformly) distributed 
in a subinterval around the strike $K$ of the put or call option, to achieve greater accuracy in the neighborhood of $K$, where the pay-off function is non-smooth; (Chan, 2010) uses a sophisticated adaptive scheme due to (Driscoll and Heryudono, 2007), for the same reason.

We already noted that for $\varphi$ we will take Hardy's multi-quadric (22). For the choice of shape parameter we follow (Fausshauer et al., 2004a;b; Hon and Mao, 1999) and put $c=4 h$. Note that since $\varphi_{c}(x)=c \varphi_{1}(x / c)$, this amounts to stationary interpolation, modulo a trivial multiplicative factor of $c=4 h$. Also note that $\varphi_{4 h}\left(x_{i}^{h}-x_{j}^{h}\right)=\varphi_{4 h}((i-j) h)=4 h \varphi_{1}((i-j)$, so that in step 2 of our algorithm we only need to invert $\Phi_{X}$ with $\varphi=\varphi_{1}$ and $X=\{0, \ldots, N\}$.

As far as we are aware, no general theoretical results are available for convergence and stability of this type of RBF-scheme. In the next section we report on a set of numerical experiments for a wide range of VG and CGMY processes, including unbounded variation processes, finding evidence of a convergence rate of 2 (for the range of $N$ 's considered), including for highly singular Lévy measures. Before turning to these numerical results, we first formally describe our RBF-scheme for the American put.

\subsection{American Put Options}

As explained in section 2.3, the idea is to successively solve the ODE on a sequence of small time-intervals $\left[\tau_{m-1}, \tau_{m}\right]$ and at the end of each time interval to compare the solution with the pay-off value at $\tau_{m}$, thereby at the same time constructing the initial value for the next time interval. This leads to the following algorithm (assuming as before that $\boldsymbol{\Phi}_{X}$ is invertible for any $X)$.

1. Choose a computational range $\left[x_{\min }, x_{\max }\right]$ in log-price space, interpolation points $X=$ $\left\{x_{0}, x_{1}, \ldots, x_{N}\right\} \subset\left[x_{\min }, x_{\max }\right]$ and a partition $\mathcal{T}:=\left\{\tau_{0}=0<\tau_{1}<\cdots<\tau_{M}=T\right\}$ of $[0, T]$. Put $\boldsymbol{x}:=\left(x_{1}, \ldots, x_{N}\right)$.

2. Choose an $\operatorname{RBF} \varphi$ and compute the matrices $\boldsymbol{\Phi}_{X}$ and $(\mathbf{L} \boldsymbol{\Phi})_{X}$ from (21) and (28), using lemma 3.1 if the Lévy measure is singular in 0 .

3. Compute the RBF-interpolant $\sum_{j} \rho_{j}(0) \varphi\left(x-x_{j}\right)$ of the initial value $g(x)=G\left(e^{x}\right), G$ being the pay-off function, and put $\boldsymbol{\rho}(0)=\left(\rho_{0}(0), \ldots, \rho_{N}(0)\right)$.

4. For $m=0,1, \cdots, M-1$, recursively compute vectors $\boldsymbol{\rho}(m+1)=\left(\rho_{0}(m+1), \ldots\right.$, $\left.\rho_{N}(m+1)\right)$ and $\boldsymbol{U}(m+1)=\left(U_{0}(m+1), \ldots, U_{N}(m+1)\right)$ according to:

(i) $\boldsymbol{\rho}(m+1)$ is the solution at time $\tau_{m+1}$ of the system (29) with initial value $\boldsymbol{\rho}(m)$ at time $\tau_{m}$;

(ii)

$$
\boldsymbol{U}(m+1):=\max \left(K-e^{\boldsymbol{x}}, \boldsymbol{\Phi}_{X} \boldsymbol{\rho}(m+1)\right),
$$

where $e^{\boldsymbol{x}}:=\left(e^{x_{1}}, \ldots, e^{x_{N}}\right)$ and where the max of two vectors is defined as the vector of the component-wise maxima;

(iii) Redefine $\boldsymbol{\rho}(m+1)$ by putting

$$
\boldsymbol{\rho}(m+1):=\boldsymbol{\Phi}_{X}^{-1} \mathbf{U}(m+1) .
$$

5. Finally, $U(x, T):=U_{X, \mathcal{T}}(x, T):=\sum_{j} \rho_{j}(M) \varphi\left(x-x_{j}\right)$.

$U_{X, \mathcal{T}}(x, T)$ is the RBF-approximation of the American put-value at log-price $x$ and timeto-maturity $T$, using the interpolation set $X$ and the time-partition $\mathcal{T}$.

Note that $\boldsymbol{\Phi}_{X} \boldsymbol{\rho}(m+1)$, with $\boldsymbol{\rho}(m+1)$ as in 4.(i), is the vector whose $i$-th component is $\sum_{j} \rho_{j}(m+1) \varphi\left(x_{i}-x_{j}\right)$, which is the value in $x_{i}$ of the RBF-approximation of the solution, 
at time $\tau_{m+1}$, of the Cauchy problem with initial value $\sum_{j} \rho_{j}(m) \varphi\left(x-x_{j}\right)$ at time $\tau_{m}$. This corresponds to the continuation value of the option when time-to-maturity is $\tau_{m+1}$, and which we then compare with the exercise value in each of the points $x_{i}$, taking the point-wise maximum in (36). This explains (36); equation (37) extracts a new $\boldsymbol{\rho}(m+1)$ which is used as initial value for the system (29) on the next time-interval. We continue in this way until we have reached $\tau_{M}=T$.

We will use a simple equally-spaced set of time-points $\tau_{m}=m k$, with $k:=T / M$ and $m \in\{0,1, \ldots, M\}$. Alternatively, one can try and construct adaptive schemes to achieve higher accuracy with less time-steps, but this is outside of the scope of the present paper. The integration of the system (29) from $\tau_{m}$ to $\tau_{m+1}$ will, as before, be done using a stiff ODE solver from matlab. In the next section we present numerical evidence that this scheme is convergent of order 2 in $N$ and order 1 in $M$.

\section{Numerical Results}

We tested our RBF algorithm by computing American and European put and call prices under a number of CGMY-processes, including unbounded variation ones $(Y>1)$. To assess accuracy, we compared the RBF-prices with prices computed, to high accuracy, using the Fourier Space Time-stepping or FST algorithm of (Jackson et al., 2008) ${ }^{1}$. For the VG model $(Y=0)$, we used the explicit solution found in (b. Madan et al., 1998) as a comparison. In our numerical experiments, we took the strike $K=1$ and the computational domain $\left[x_{\min }, x_{\max }\right]=[-9,9]$, corresponding to a comfortably large price range of $\left[e^{-9}, e^{9}\right]$ for the price of the underlying. We computed RBF prices $U_{N}(x, T)$ using equally spaced interpolation points $X_{N}$ as in (35), with $N$ varying from 100 to 3600 , increasing by steps of 500. The RBF algorithms of subsection 3.4 and 3.5 were implemented in MATLAB R2007b, using quadgk to compute the integrals (33) and (34) and ode15s to solve the ODE (29). The FST reference prices were computed by truncating to the same log-price interval, [-9,9], as used for the RBF scheme, and applying the FFT with $N=32768$ nodes, which gave a 6 -decimal point accuracy or better in (Jackson et al., 2008).

The RBF-solution was compared with the reference solution $V(x, T)$ (in $\log$-price coordinates) on a set of $N_{\text {eval }}$ equidistant evaluation points

$$
\widehat{x}_{i}:=\widehat{x}_{i}^{\widehat{h}}:=\widehat{x}_{\min }+j \widehat{h}, \quad j=0, \ldots, N_{\text {eval }},
$$

where $\left[\widehat{x}_{\min }, \widehat{x}_{\max }\right] \subset\left(x_{\min }, x_{\max }\right)$ is a, typically small, subinterval and $\widehat{h}=\left(\widehat{x}_{\max }-\widehat{x}_{\min }\right) / N_{\text {eval }}$, in both the sup-norm

$$
E_{\infty}(N):=\max _{0 \leq i \leq N_{\text {eval }}}\left|U_{N}\left(\widehat{x}_{i}, T\right)-V\left(\widehat{x}_{i}, T\right)\right|,
$$

and the, smooth, mean square norm,

$$
E_{2}(N):=\sqrt{\frac{1}{N_{\text {eval }}} \sum_{i=1}^{N_{\text {eval }}}\left(U_{N}\left(\widehat{x}_{i}, T\right)-V\left(\widehat{x}_{i}, T\right)\right)^{2}} .
$$

Given that, for option trading, the regions of principal interest where to evaluate option prices are typically those near to the strike, $\left[\widehat{x}_{\min }, \widehat{x}_{\max }\right]$ should contain the log-strike, 0 , and can be taken much smaller than the interpolation (or collocation) range $\left[x_{\min }, x_{\max }\right]$;

${ }^{1}$ For European options, the FST-algorithm is a variant of Carr and Madan's FFT algorithm, (Carr and Madan, 1999), with the Fourier transform of the pay-off being computed numerically using the FFT, instead of being evaluated analytically; the American problem is reduced to a sequence of European problems using a time-discretisation, similar to what is done here; (Jackson et al., 2008) also consider other path-dependent options. 
in this context, (Pettersson et al., 2008) refer to (38) as the financial error-norm. We took $\left[\widehat{x}_{\min }, \widehat{x}_{\max }\right]=[\log (0.05), \log 2]$, corresponding to a price range of $[0.05,2]$ around the strike of 1 , and $N_{\text {eval }}$ equal to 1950 , so that the error is evaluated on a comparatively fine grid with a spacing of less than 0.002 .

Remark 1: According to (Cont and Voltchkova, 2005, prop. 4.1), the localisation error on $\left[\widehat{x}_{\min }, \widehat{x}_{\max }\right]$ caused by localising the pay-off to $\left[x_{\min }, x_{\max }\right]=[-9,9]$ is exponentially small in the distance $\min \left(x_{\max }-\widehat{x}_{\max },\left|x_{\min }-\widehat{x}_{\min }\right|\right)$ and is therefore expected to be negligible (an observation which is relevant for both the RBF and to the FST schemes). This was confirmed numerically by increasing $x_{\max }=-x_{\min }$ while keeping $\left[\widehat{x}_{\min }, \widehat{x}_{\max }\right]=[\log (0.05), \log 2]$ fixed.

The convergence rate $R_{E}$ of our RBF-scheme is estimated from $E(N) \simeq C N^{-R_{E}}$, where $E=E_{\infty}$ or $E=E_{2}$. Thus, $R_{E}=\log \left(E\left(N_{1}\right) / E\left(N_{2}\right)\right) / \log \left(N_{2} / N_{1}\right)$, where $N_{1}$ and $N_{2}$ are two successive choices of number of interpolation points. In the tables below, we will write $R_{\infty}$ if we use the sup-norm and $R_{2}$ if we use the mean square norm.

Tables 1 to 4 of appendix B illustrate the performance of the RBF algorithm for computing European call and put prices under CGMY processes with $Y$ ranging from 0 (VarianceGamma) to 1.6. In all cases, we numerically found a convergence rate of 2 , valid over the range of $N$ 's considered. This is the same rate as found by (Jackson et al., 2008) for the FTS method. We note, though, that our present implementation of the RBF method underperforms FST in terms of execution time. This is mostly on account of the adaptive ODE solver we used, which though very precise, considerably slows down the computation. Using a simpler ODE scheme could improve performance. Since our main focus in this paper was on the discretisation error in $x$, we have left such improvements for future work.

A convergence rate of 2 is also found for the best performing FD methods, at least for finite variation processes $(Y<1)$, though not for processes of unbounded variation: cf. (Wang et al., 2007). The paper of (Almendral and Oosterlee, 2007a) achieves second order convergence for finite variation processes using reduction to weakly singular Volterra equations, a method which for the moment does not seem to apply to infinite variation processes.

As mentioned in section 3.1, RBF interpolation can sometimes achieve so-called spectral rates of approximation on compact sets, that is, approximation errors which are smaller than any power of $d$, or even smaller than $\exp (-c / d)$ for some $c>0$, where $d$ is the "filling distance" of the set of interpolation points, which in the case of equidistant points (35), is simply $h$; cf. (Buhmann, 2003; Wendland, 2005) and their references. One might therefore have hoped for a better rate of convergence of the RBF scheme. Note, however, that spectral convergence only occurs with non-stationary RBF-interpolation. For stationary interpolation, which was used here, an algebraic convergence rate is the norm: cf. (Buhmann, 2003). In (Pettersson et al., 2008), the authors also find algebraic convergence rates when letting the shape parameter $c$ of the multi-quadric vary with the interpolating set (they let $c \simeq h^{p}$ for $p=1 / 2,3 / 4$ or 1 , the stationary case, in a numerical example).

We next examined whether our approximate RBF solution can be used to compute the option's Greeks, in particular whether its $\Delta$ and $\Gamma$ are well approximated by the respective derivatives of $U_{N}(x, \tau)$ with respect to $S=e^{x}$ :

$$
\Delta_{N}(x):=\frac{\partial U_{N}(x, \tau)}{\partial S}=\sum_{j=1}^{N} \rho_{j}(\tau) \frac{\partial \varphi\left(x-x_{j}\right)}{\partial x} e^{-x}
$$

and

$$
\Gamma_{N}(x)=\frac{\partial^{2} U_{N}(x, \tau)}{\partial S^{2}}=\sum_{j=1}^{N} \rho_{j}(\tau) \frac{\partial^{2} \varphi\left(x-x_{j}\right)}{\partial x^{2}} e^{-2 x}-\Delta_{N} e^{-x}
$$


Testing these expressions (with $N=3600$ ) against the $\Delta$ and $\Gamma$ computed by finite differences from the FST solution or Madan, Carr and Chang's explicit solution, we found excellent agreement. See figure B1 for an example.

Tables B5 to B9 in Appendix B gives some of our results for the performance of the RBFalgorithm for American puts. The reference solution was in all cases computed using the FST algorithm. Note that at each stage we quadrupled the number of intermediary time steps ${ }^{1} M_{0}$ while doubling the number of space-steps $N$. The results of tables B5 to B9 are compatible with second order convergence in $N$ and first-order convergence in $M_{0}$ but do not directly show this. We therefore also investigated convergence with rspect to $M_{0}$ and $N$ seperatedly, and indeed found first order convergence in time and second order convergence in space, over the range of $M_{0}$ and $N$ considered. Table B10 gives an example: further results are available from the authors.

For the remainder of this section, we set $N=3600$ and $M_{0}=2560$. Figure B2 plots an example of the option $\Delta$ and $\Gamma$ obtained using the RBF-scheme, with an $Y>1$. Figure B3 shows the sensitivity of option prices and free boundary with respect to the $Y$-parameter, computed using the RBF algorithm, following (Almendral, 2004), and extending the latter to the $Y \geq 1$ range.

As a final test and illustration of our algorithm, we numerically investigate the smooth pasting principle, following (Almendral, 2004) and (Almendral and Oosterlee, 2007b). The smooth pasting or smooth fit principle states that the $\Delta$ of an American option is continuous across the exercise boundary. Equivalently, for an American put, the option value's right derivative with respect to the underlying has to be equal to -1 at the exercise boundary (the right- and left derivative both exist since the value function is a convex function). The smooth pasting principle is known to hold for infinite variation Lévy models, but can fail for bounded variation processes: see e.g. (Boyarchenko and Levendorskiï, 2002b), (Alili and Kyprianou, 2005) for perpetual puts, and (Almendral and Oosterlee, 2007b; Lamberton and Mikou, 2012) for the finite maturity case. Figure B4 recomputes an example from (Almendral, 2004) (but with a different strike $K$ ) using the RBF scheme, illustrating the possibility of both non-smooth and smooth fit in the bounded variation case. Figure B5 illustrated the validity of the smooth fit principle in the infinite variation case $(Y \in[1,2))$, at least to the accuracy provided by the RBF algorithm. We note the absence of oscillations close to the jump in the $\Delta$ in figure B4, contrary to what can happen with a FD algorithm: see (Almendral and Oosterlee, 2007b).

\section{Discussion}

We investigated a simple scheme based on RBF interpolation for numerically solving the pricing PIDE of exponential Lévy models. The scheme was numerically found to be second order convergent for examples of infinite activity Lévy processes from the CGMY-KoBoL class, including processes of unbounded variation. On this evidence, the RBF scheme performs comparably to Fourier transform-based schemes such as the FST, and better than current FD schemes, who to date only achieve second order convergence for Lévy processes of bounded variation. Contrary to FD methods, no special discretisation procedures or diffusion approximations near the singularity of the Lévy measure are needed, since only the action of the PIDE on the basis function has to be computed, and the relevant integral can be explicitly desingularized. This makes the RBF scheme simple to implement.

\footnotetext{
${ }^{1}$ We use $M_{0}$ to avoid confusion with the $M$-parameter in CGMY.
} 
A disadvantage of the RBF scheme is that it requires the numerical solution of a full linear system, which in practice limits the number of interpolation points the scheme can handle. On the other hand, fewer points are needed to achieve good accuracy than with FST or FD. We also note that the best performing FD method, that of (Wang et al., 2007), is a fully implicit scheme leading to non-sparse systems also.

The second order convergence has only been shown in numerical experiments, and the challenge remains to find a rigorous proof. This is currently an active area for research. We note in passing that the absence of theoretical justification is shared with other numerical methods which have been proposed in this area, such as (Jackson et al., 2008) or (Almendral and Oosterlee, 2007a)): it is only for FD methods that a complete stability and convergence analysis has been given to this date; cf. (Wang et al., 2007) and its references.

A main potential of the RBF method lies in its applicability to multi-dimensional models and the possibility of a mesh-free implementation. The present scheme can be straightforwardly generalized to higher dimensional Lévy models, including its handling of the singularity of the Lévy measure. This paper limited itself to one-dimensional problems in order to asses the accuracy of the RBF scheme and compare its performance with other existing methods, as a necessary preliminary to attacking the multi-dimensional case. The scheme does have an independent interest even in the one-dimensional case, for fast calibration and, potentially, for option pricing in non-parametric Lévy models.

\section{Acknowledgment}

This paper is based on the PhD thesis of the second-named author. The authors would like to express their thanks to Brad Baxter, Simon Hubbert, Elisabeth Larsson and William Shaw for their comments and advice, as well as to the two anonymous referees for their carefull reading of the manuscript and their thoughtful comments.

\section{References}

Achdou, Y. and Pironneau, O. (2010) Computational methods for quantitative finance: Finite Element methods for derivative pricing. In: R. Cont (Ed.) Encyclopedia of Quantitative Finance, pp. 491-514 (John Wiley \& Sons Ltd., England).

Alili, L. and Kyprianou, A. E. (2005) Some remarks on first passage of Lévy processes, the American put and pasting principles, Ann. Appl. Probab., 15, pp. 2062-2080.

Almendral, A. (2004) Numerical Valuation of American Options under the CGMY Process. In: W. Schoutens, A. Kyprianou and P. Wilmott (Eds.) Exotic Option Pricing and Advanced Lévy Models (UK: Wiley).

Almendral, A. and Oosterlee, C. (2005) Numerical Valuation of Options with Jumps in the Underlying, Applied Numerical Mathematics, 53, pp. 1-18.

Almendral, A. and Oosterlee, C. W. (2007a) Accurate Evaluation of European and American Options Under the CGMY Process, SIAM Journal on Scientific Computing, 29, pp. 93-117.

Almendral, A. and Oosterlee, C. W. (2006) Highly Accurate Evaluation of European and American Options under the Variance Gamma Process, Journal of Computational Finance, 10, pp. 21-42.

Almendral, A. and Oosterlee, C. W. (2007b) On American Options under the Variance Gamma Process, Applied Mathematical Finance, 14, pp. $131-152$.

Andersen, L. and Andreasen, J. (2000) Jump-diffusion Processes: Volalitility Smile fitting and Numerical Methods for Option Pricing, Review of Derivatives Research, 4, pp. 231-262.

Applebaum, D. (2004) Lévy Processes and Stochastic Calculus, (Cambridge University Press, Cambridge, UK).

b. Madan, D., p. Carr, P. and Chang, E. C. (1998) The Variance Gamma Process and Option Pricing, European Finance Review, 2, pp. 79-105.

Bertoin, J. (1996) Lévy Processes, (Cambridge University Press, Cambridge UK).

Boyarchenko, S. I. and Levendorskii, S. Z. (2002a) Non-Gaussian Merton-Black-Scholes Theory, Advanced Series on Statistical Science \& Applied Probability Vol. 9 (River Edge, NJ: World Scientific Publishing Co. Inc.).

Boyarchenko, S. I. and Levendorskii, S. Z. (2002b) Perpetual American options under Lévy processes, SIAM Journal on Control and Optimization, 40, pp. 1663-1696.

Buhmann, M. and Dyn, N. (1993) Spectral convergence of multiquadric interpolation, Proceedings of the Edinburgh Mathematical Society, 36, pp. 319 - 333.

Buhmann, M. D. (2003) Radial Basis Functions: Theory and Implementations, Cambridge Monographs on Applied and Computational Mathematics Vol. 12 (Cambridge: Cambridge University Press). 
Carr, P., Geman, H., Madan, D. B. and Yor, M. (2002) The Fine Structure of Asset Returns: An Empirical Investigation, Journal of Business, 75, pp. 305-332.

Carr, P. and Madan, D. B. (1999) Option Valuation Using the Fast Fourier Transform, Journal of Computational Finance, 2, pp. 61-73.

Carr, P., Madan, D. B. and Chang, E. C. (1998) The Variance Gamma Process and Option Pricing, European Finance Review, 2, pp. 79-105.

Chan, R. T. L., Pricing Options under Jump-Diffusion Models by Adaptive Radial Basic Functions. (2010) , Technical report, Birkbeck, University of London, U.K See http://opus.bath.ac.uk/19329/.

Chan, R. T. L., Options Pricing under the One-Dimensional Jump-diffusion Model using the Radial Basis Function Interpolation Scheme. (2011), Technical report, UEL Royal Docks Business School, University of East London, U.K To appear in Review of Derivatives Research.

Cont, R. and Tankov, P. (2004) Financial Modelling With Jump Processes, Chapman \& Hall/CRC Financial Mathematics Series (Boca Raton, Fla., London: Chapman \& Hall/CRC).

Cont, R. and Voltchkova, E. (2005) A Finite Difference Scheme for Option Pricing in Jump Diffusion and Exponential Lévy Models, SIAM Journal on Numerical Analysis, 43, pp. 1596-1626.

d'Halluin, Y., Forsyth, P. and Vetzalz, K. (2005) Robust Numerical Methods for Contingent Claims under Jump Duffusion Process, IMA J. Num. Anal., 25, pp. 87-112.

d'Halluin, Y., Forsyth, P. A. and Labahn, G. (2004) A Penalty Method for American Options with Jump Diffusion Processes, Numerische Mathematik, 97, pp. 321-352.

Driscoll, T. A. and Heryudono, A. R. H. (2007) Adaptive Residual Subsampling Methods for Radial Basis Function Interpolation and Collocation Problems, Comput. Math. Appl., 53, pp. 927-939.

Eberlein, E. (2001) Application of Generalized Hyperbolic Lévy Motion to Finance. In: O. E. Barndorff-Nielsen, T. Mikosch and S. Resnick (Eds.) Lévy Processes-Theory and Application (Boston: Birkhäuser).

Fasshauer, G. E. and McCourt, M. J. (2012) Stable Evaluation of Gaussian Radial Basis Function Interpolants, SIAM J. Sci. Comput., 34(2), pp. 737-762.

Fasshauer, G. E. and Zhang, J. G. (2007) On Choosing Optimal Shape Parameters for RBF Approximation, Numerical Algorithms, 45, pp. 345-368.

Fasshauer, G. E. (2007) Meshfree Approximation Methods with MATLAB, Interdisciplinary Mathematical Sciences Vol. 6 (World Scientific Sciences, Hackensack, New Jersey).

Fausshauer, G. E., Khaliq, A. Q. M. and Voss, D. A. (2004a) A Parallel Time Stepping Approach Using Meshfree Approximations for Pricing Options with Non-smooth Payoffs. In: Proceedings of Third World Congress of the Bachelier Finance Society, Chicago, 2004, July.

Fausshauer, G. E., Khaliq, A. Q. M. and Voss, D. A. (2004b) Using Meshfree Approximation for Multi-Asset American Option Problems, J. Chinese Institute Engineers, 27, pp. $563-571$.

Fornberg, B. and Wright, G. (2004) Stable Computation of Multiquadric Interpolants for All Values of the Shape Parameter, Comput. Math. Appl., 47, pp. 497-523.

Fornberg, B., Larsson, E. and Flyer, N. (2011) Stable Computations with Gaussian Radial Basis Functions, SIAM J. Scientific Computing, 33(2), pp. 869-892.

Hilber, N., Reichmann, O., Schwab, C. and Winter, C. (2013) Computational Methods for Quantitative Finance: Finite Element Methods for Derivative Pricing, (Springer, Heidelberg - New York - Dordrecht - London: Springer Finance).

Hirsa, A. and Madan, D. B. (2004) Pricing American Options Under Variance Gamma, Journal of Computational Finance, 7 , pp. 11-22.

Hon, Y. C. and Mao, X. Z. (1999) A Radial Basis Function Method for Solving Options Pricing Model, Financial Engineering, 8, pp. 31-49.

Hull, J. (2012) Options, Futures, and Other Derivatives, 8, (Pearson / Prentice Hall).

Iserles, A. (2009) A First Course in the Numerical Analysis of Differential Equations, Cambridge Texts in Applied Mathematics Vol. 43 (Cambridge University Press, Cambridge, UK).

Jackson, K. R., Sebastian, J. and Vladimir, S. (2008) Fourier Space Time-stepping for Option Pricing with Lévy Models, the Journal of Computational Finance, 12, pp. 1-29.

Kansa, E. J. and Carlson, R. E. (1992) Improved Accuracy of Multiquadric Interpolation Using Variable Shape Parameters, Comput. Math. Applic., 24, pp. 99-120.

Koponen, I. (1995) Analytic Approach to the Problem of Convergence of Truncated Lévy Flights Towards the Gaussian Stochastic Process, Physical Review E, 52, pp. 1197-1199.

Korn, R. and Rogers, L. C. G. (2005) Stocks paying discrete dividend: modelling and option pricing, J. of Derivatives, 13(2), p. 4448.

Kudryavtsev, O. and Levendorskii, S. (2009) Fast and accurate pricing of barrier option under Lévy processes, FINANCE AND STOCHASTICS, 13, pp. 531-562.

Lamberton, D. and Mikou, M. (2012) The smooth-fit property in an exponential Lvy model, Journal of Applied Probability, 49, pp. 137-149.

Larsson, E., Åhlander, K. and Hall, A. (2008) Multi-dimensional Option Pricing Using Radial Basis Functions and the Generalized Fourier Transform, Journal of Computational and Applied Mathematics, 222, pp. 175-192.

Levendorskii, S. (2004) Early exercise boundary and option pricing in Levy driven models, Quantitative Finance, 4, pp. $525-547$.

Madan, D. B. and Milne, F. (1991) Option Pricing with V. G. Martingale Components, Mathematical Finance, 1, pp. 39-55.

Madan, D. B. and Seneta, E. (1990) The Variance Gamma (V.G.) Model for Share Market Returns, Journal of Business, 63, pp. 511-524.

Madych, W. R. (1992) Miscellaneous error bounds for multiquadric and related interpolators, Comput. Math. Appl., 24, pp. 121-138.

Matache, A. M., Nitsche, P. A. and Schwab, C. (2005a) Wavelet Galerkin Pricing of American Options on Lévy Driven Assets, Journal of Quantitative Finance, 5, pp. $403-424$.

Matache, A. M., Schwab, C. and Wihler, T. P. (2005b) Fast Numerical Solution of Parabolic Integrodifferential Equations with Applications in Finance, SIAM Journal on Scientific Computing, 27, pp. 369 - 393. 
Merton, R. C. (1976) Option Pricing When Underlying Stock Returns are Discontinuous., Journal of Financial Economics, 3, pp. 125-144.

Pettersson, U., Larsson, E., Marcusson, G. and Persson, J. (2008) Improved Radial Basis Function Methods for Multidimensional Option Pricing, Journal of Computational and Applied Mathematics, 222, pp. 82-93.

Peškir, G. and Shiryaev, A. (2006) Optimal Stopping and Free-Boundary Problems, (Birkäuser Verlag, Basel - Boston Berlin).

Revuz, D. and Yor, M. (1999) Continuous Martingales and Brownian motion, third, (Springer, Berlin - London).

Sato, K. I. (1999) Lévy Processes and Infinitely Divisible Distributions, (Cambridge University Press, Cambridge UK).

Schaback, R. (1994) Error Estimates and Condition Numbers for Radial Basis Function Interpolation, Adv. Comput. Math, 3, pp. 251-264.

Schaback, R. (1995) Multivariate Interpolation and Approximation by Translates of a Basis Function. In: C. Chui and L. Schumaker (Eds.) Approximation Theory VIII, Vol. 1: Approximation and Interpolation, pp. 491-514 (World Scientific Publishing Co., Inc).

Schoutens, W. (2003) Lévy Processes in Finance : Pricing Financial Derivatives, Wiley Series in Probability and Mathematical Statistics (Chichester : Wiley).

Shampine, L. F. (2008) Vectorized Adaptive Quadrature in MATLAB, Journal of Computational and Applied Mathematics, 211, pp. 131-140.

Tankov, P. and Voltchkova, E., Jump-Diffusion Models: A Practitioners Guide. (2009), Technical report, Universit Paris-Diderot (Paris 7), France See http://people.math.jussieu.fr/ tankov/tankov_voltchkova.pdf.

Wang, I. R., Wan, J. W. L. and Forsyth, P. A. (2007) Robust Numerical Valuation of European and American Options under the CGMY Process, Journal of Computational Finance, 10, pp. 31-69.

Wendland, H. (2005) Scattered Data Approximation,, Cambridge Monographs on Applied and Computational Mathematics Vol. 17 (Cambridge: Cambridge University Press).

Zhang, X. L. (1997) Numerical Analysis of American Option Pricing in a Jump-diffusion Model, Mathematics of Operations Research, 22 , pp. $668-690$.

\section{Appendix A. Modified Algorithm with Conditionally Definite Radial Basis Functions}

Let $\mathbb{P}_{n}^{k}$ denote the finite-dimensional vector space of polynomials of degree at most $k$ on $\mathbb{R}^{n}$. A function $\varphi: \mathbb{R}^{n} \rightarrow \mathbb{R}$ is called (strictly) conditionally positive definite of order $k$ (Buhmann, 2003; Wendland, 2005, cf.) if, for any finite set of points $X=\left\{x_{1}, \ldots, x_{N}\right\}$, the quadratic form $\sum_{i, j} \varphi\left(x_{i}-x_{j}\right) \alpha_{i} \alpha_{j}$ is (strictly) positive definite on the linear subspace of all $\alpha=\left(\alpha_{1}, \ldots, \alpha_{N}\right) \in \mathbb{R}^{N}$ such that

$$
\sum_{j=1}^{N} \alpha_{j} p\left(x_{j}\right)=0, \quad \forall p \in \mathbb{P}_{n}^{k-1} .
$$

Examples are the generalized multi-quadrics $\left(|x|^{2}+c^{2}\right)^{k / 2}, k>0$, which are of order $\max (0,\lceil k\rceil)$; in particular, the multi-quadric is of order 1 .

Given a conditionally positive definite radial basis function $\varphi$, of order $k$, the associated RBF-interpolants are now of the form

$$
s_{X}(x)=\sum_{j=1}^{N} \alpha_{j} \varphi\left(x-x_{j}\right)+\sum_{k=1}^{Q} \beta_{k} p_{k}(x),
$$

where $\left\{p_{k}: 1 \leq \nu \leq Q\right\}, Q:=\operatorname{dim}\left(\mathbb{P}_{n}^{k-1}\right)$, is some basis of $\mathbb{P}_{n}^{k-1}$, and where the coefficients $\alpha_{j}$ and $\beta_{k}$ are now determined by the augmented linear system

$$
\left\{\begin{array}{l}
s_{X, \varphi}\left(x_{i}\right)=f\left(x_{i}\right), 1 \leq i \leq N \\
\sum_{j=1}^{N} \alpha_{j} p_{k}\left(x_{j}\right)=0, \quad 1 \leq k \leq Q .
\end{array}\right.
$$

where $f=f(x)$ is the function which is to be interpolated. The unique solvability of this system is an easy consequence of the conditional definiteness of $\varphi$, if we moreover assume $X$ to be insolvent for $\mathbb{P}_{n}^{k-1}$ (meaning that the only polynomial of degree at most $k-1$ vanishing on $X$ is the zero polynomial): cf. (Buhmann, 2003), (Wendland, 2005). 
It is now natural to modify our Ansatz (24) as follows: given a $\varphi$ which is conditionally strictly definite of order $k$, and a set of collocation points $X=\left\{x_{1}, \ldots, x_{N}\right\}$, univalent for $\mathbb{P}_{n}^{k-1}$, we put

$$
U_{X}(x, \tau):=\sum_{j=1}^{N} \rho_{j}(\tau) \varphi\left(x-x_{j}\right)+\sum_{k=1}^{Q} \gamma_{k}(\tau) p_{k}(x),
$$

and require that $U_{X}$ satisfies the augmented system

$$
\left\{\begin{array}{l}
\partial_{\tau} U_{X}\left(x_{i}, \tau\right)=\mathcal{L}\left[U_{X}\right]\left(x_{i}, \tau\right), \quad 1 \leq i=1, \ldots, N \\
\sum_{j=1}^{N} \frac{d \rho_{j}}{d \tau} p_{k}\left(x_{j}\right)=0, \quad k=1, \ldots, Q
\end{array}\right.
$$

for all $\tau \geq 0$. This then leads to the following system of ODEs for $\rho(\tau)=\left(\rho_{1}(\tau), \ldots, \rho_{N}(\tau)\right)$ and $\gamma(\tau)=\left(\gamma_{1}(\tau), \ldots, \gamma_{Q}(\tau)\right)$ : defining $\boldsymbol{\Phi}_{X}$ and $(\mathbf{L} \boldsymbol{\Phi})_{X}$ as before (cf. (21) and (28)), but now also introducing the $N \times Q$ matrices

$$
\mathbf{P}_{X}:=\left(p_{k}\left(x_{j}\right)\right) \begin{gathered}
\substack{1 \leq j \leq N \\
1 \leq k \leq Q} \\
1 \leq k
\end{gathered}
$$

and

$$
(\mathbf{L P})_{X}:=\left(\mathcal{L}\left[p_{k}\right]\left(x_{j}\right)\right)_{\substack{1 \leq j \leq N \\ 1 \leq k \leq Q}}
$$

where $j$ is the row-index, we now find that

$$
\left(\begin{array}{cc}
\mathbf{\Phi}_{X} & \mathbf{P}_{X} \\
\mathbf{P}_{X}^{t} & 0
\end{array}\right)\left(\begin{array}{c}
\frac{d}{d \tau} \rho(\tau) \\
\frac{d}{d \tau} \gamma(\tau)
\end{array}\right)=\left(\begin{array}{cc}
(\mathbf{L} \mathbf{\Phi})_{X} & (\mathbf{L P})_{X} \\
0 & 0
\end{array}\right)\left(\begin{array}{l}
\rho(\tau) \\
\gamma(\tau)
\end{array}\right)
$$

The coefficient matrix of the time-derivative on the right is non-singular. The system (A5) is to be completed by the initial condition

$$
\left(\begin{array}{cc}
\mathbf{\Phi}_{X} & \mathbf{P}_{X} \\
\mathbf{P}_{X}^{t} & 0
\end{array}\right)\left(\begin{array}{l}
\rho(0) \\
\gamma(0)
\end{array}\right)=\left(\begin{array}{c}
\left.g\right|_{X} \\
0
\end{array}\right)
$$

were, as before, $\left.g\right|_{X}$ is the vector $\left(g\left(x_{1}\right), \ldots, g\left(x_{N}\right)\right)$. We now of course require that $\mathcal{L}\left[p_{k}\right]$ is well-defined, that is, that $\mathcal{L}$ acts on polynomials of degree less than $k$. This poses no problem if the Lévy-measure is exponentially decreasing (or, more generally, if all polynomials in $\mathbb{P}_{n}^{k-1}$ are integrable with respect to the Lévy measure), as is the case for the CGMY model.

\section{Appendix B. Numerical results: tables and figures}




\begin{tabular}{ccccc}
\hline \hline $\mathrm{N}$ & $E_{\infty}(N)$ & $R_{\infty}$ & $E_{2}(N)$ & $R_{2}$ \\
\hline \hline 100 & $1.250133 \mathrm{E}-02$ & N/A & $2.860813 \mathrm{E}-03$ & N/A \\
600 & $3.746827 \mathrm{E}-04$ & 1.958 & $6.041865 \mathrm{E}-05$ & 2.153 \\
1100 & $1.082384 \mathrm{E}-04$ & 2.049 & $1.759146 \mathrm{E}-05$ & 2.036 \\
1600 & $5.049619 \mathrm{E}-05$ & 2.035 & $8.271495 \mathrm{E}-06$ & 2.014 \\
2100 & $2.912316 \mathrm{E}-05$ & 2.024 & $4.791782 \mathrm{E}-06$ & 2.008 \\
2600 & $1.894538 \mathrm{E}-05$ & 2.013 & $3.122765 \mathrm{E}-06$ & 2.005 \\
3100 & $1.331338 \mathrm{E}-05$ & 2.006 & $2.195320 \mathrm{E}-06$ & 2.003 \\
3600 & $9.878318 \mathrm{E}-06$ & 1.996 & $1.627210 \mathrm{E}-06$ & 2.003 \\
\hline \hline
\end{tabular}

Table B1. $\quad E_{\infty}$ and $E_{2}$ for the RBF approximation of a European put with strike $K=1$ and maturity $T=0.13972$ under a VG model with parameters $\sigma=0.17875, \nu=0.13317$ and $\theta=-0.30649$ and with $r=0.0533$ and $q=0.011$. The parameters were taken from Hirsa and Madan (2004) and the reference solution was computed using the explicit formula of (b. Madan et al., 1998).

\begin{tabular}{ccccc}
\hline \hline $\mathrm{N}$ & $E_{\infty}(N)$ & $R_{\infty}$ & $E_{2}(N)$ & $R_{2}$ \\
\hline \hline 100 & $1.007298 \mathrm{E}-03$ & N/A & $7.105950 \mathrm{E}-04$ & N/A \\
600 & $2.728381 \mathrm{E}-05$ & 2.014 & $1.932604 \mathrm{E}-05$ & 2.012 \\
1100 & $8.112433 \mathrm{E}-06$ & 2.001 & $5.740643 \mathrm{E}-06$ & 2.003 \\
1600 & $3.838466 \mathrm{E}-06$ & 1.997 & $2.711727 \mathrm{E}-06$ & 2.002 \\
2100 & $2.232595 \mathrm{E}-06$ & 1.993 & $1.573655 \mathrm{E}-06$ & 2.001 \\
2600 & $1.460368 \mathrm{E}-06$ & 1.987 & $1.026391 \mathrm{E}-06$ & 2.001 \\
3100 & $1.030620 \mathrm{E}-06$ & 1.982 & $7.218958 \mathrm{E}-07$ & 2.001 \\
3600 & $7.672202 \mathrm{E}-07$ & 1.974 & $5.352399 \mathrm{E}-07$ & 2.001 \\
\hline \hline
\end{tabular}

Table B2. $E_{\infty}$ and $E_{2}$ for the RBF-approximation of a European call with strike $K=1$ and maturity $T=0.25$ under a CGMY-model with $C=16.97, G=7.08, M=29.97$ and $Y=0.6442 ; r=0.06$ and $q=0$. The parameters were taken from Wang et al. (2007). The bench-mark solution was computed by the FFT method.

\begin{tabular}{ccccc}
\hline \hline $\mathrm{N}$ & $E_{\infty}(N)$ & $R_{\infty}$ & $E_{2}(N)$ & $R_{2}$ \\
\hline \hline 100 & $1.818922 \mathrm{E}-03$ & N/A & $8.933607 \mathrm{E}-04$ & N/A \\
600 & $4.801009 \mathrm{E}-05$ & 2.029 & $2.407100 \mathrm{E}-05$ & 2.017 \\
1100 & $1.426400 \mathrm{E}-05$ & 2.002 & $7.148878 \mathrm{E}-06$ & 2.003 \\
1600 & $6.745556 \mathrm{E}-06$ & 1.999 & $3.376818 \mathrm{E}-06$ & 2.002 \\
2100 & $3.922070 \mathrm{E}-06$ & 1.994 & $1.959592 \mathrm{E}-06$ & 2.001 \\
2600 & $2.564791 \mathrm{E}-06$ & 1.989 & $1.278109 \mathrm{E}-06$ & 2.001 \\
3100 & $1.809682 \mathrm{E}-06$ & 1.983 & $8.989379 \mathrm{E}-07$ & 2.001 \\
3600 & $1.346800 \mathrm{E}-06$ & 1.976 & $6.665049 \mathrm{E}-07$ & 2.001 \\
\hline \hline
\end{tabular}

Table B3. $\quad E_{\infty}$ and $E_{2}$ for the RBF approximation of a European put with strike $K=1$ and maturity $T=1$ under a CGMY model with $C=0.42, G=4.37, M=191.2$ and $Y=1.0102 ; r=0.06$ and $q=0$. The parameters were taken from Wang et al. (2007) and the reference solution was computed using FFT. 


\begin{tabular}{ccccc}
\hline \hline $\mathrm{N}$ & $E_{\infty}(N)$ & $R_{\infty}$ & $E_{2}(N)$ & $R_{2}$ \\
\hline \hline 100 & $8.046568 \mathrm{E}-04$ & N/A & $6.222927 \mathrm{E}-04$ & N/A \\
600 & $2.187699 \mathrm{E}-05$ & 2.012 & $1.693462 \mathrm{E}-05$ & 2.011 \\
1100 & $6.504920 \mathrm{E}-06$ & 2.001 & $5.030391 \mathrm{E}-06$ & 2.003 \\
1600 & $3.077629 \mathrm{E}-06$ & 1.997 & $2.376264 \mathrm{E}-06$ & 2.002 \\
2100 & $1.789899 \mathrm{E}-06$ & 1.993 & $1.379002 \mathrm{E}-06$ & 2.001 \\
2600 & $1.170574 \mathrm{E}-06$ & 1.988 & $8.994724 \mathrm{E}-07$ & 2.001 \\
3100 & $8.260453 \mathrm{E}-07$ & 1.982 & $6.326538 \mathrm{E}-07$ & 2.001 \\
3600 & $6.147193 \mathrm{E}-07$ & 1.976 & $4.691162 \mathrm{E}-07$ & 2.000 \\
\hline \hline
\end{tabular}

Table B4. $E_{\infty}$ and $E_{2}$ for the RBF approximation of a European call with strike $K=1$ and maturity $T=0.25$ under an extended CGMY model with $\sigma=0.2, C=1, G=8.8, M=9.2$ and $Y=1.6$ and with $r=0.01$ and $q=0$. The parameters were taken from Wang et al. (2007) and the reference solution was computed using FFT.

\begin{tabular}{cccccc}
\hline \hline$N$ & $M_{0}$ & $E_{\infty}\left(N, M_{0}\right)$ & $R_{\infty}$ & $E_{2}\left(N, M_{0}\right)$ & $R_{2}$ \\
\hline \hline 225 & 10 & $1.498895 \mathrm{E}-03$ & N/A & $8.246744 \mathrm{E}-04$ & N/A \\
450 & 40 & $3.749387 \mathrm{E}-04$ & 1.999 & $1.634605 \mathrm{E}-04$ & 2.335 \\
900 & 160 & $9.374599 \mathrm{E}-05$ & 2.000 & $4.072318 \mathrm{E}-05$ & 2.005 \\
1800 & 640 & $2.343723 \mathrm{E}-05$ & 2.000 & $1.000968 \mathrm{E}-05$ & 2.024 \\
3600 & 2560 & $5.859308 \mathrm{E}-06$ & 2.000 & $2.481692 \mathrm{E}-06$ & 2.012 \\
\hline \hline
\end{tabular}

Table B5. $E_{\infty}$ and $E_{2}$ for the RBF approximation of an American put with strike $K=1$ and (time-to-) maturity $T=0.25$ under a CGMY model with parameters $C=16.97, G=7.08, M=29.97$ and $Y=0.6442$, and with $r=0.06$ and $q=0$. The parameters were taken from (Wang et al., 2007), and the reference solution was computed using FST, as for the other American options below.

\begin{tabular}{cccccc}
\hline \hline$N$ & $M_{0}$ & $E_{\infty}\left(N, M_{0}\right)$ & $R_{\infty}$ & $E_{2}\left(N, M_{0}\right)$ & $R_{2}$ \\
\hline \hline 225 & 10 & $6.082331 \mathrm{E}-03$ & N/A & $3.650482 \mathrm{E}-03$ & N/A \\
450 & 40 & $1.541366 \mathrm{E}-03$ & 1.980 & $9.076575 \mathrm{E}-04$ & 2.008 \\
900 & 160 & $3.832039 \mathrm{E}-04$ & 2.008 & $2.281262 \mathrm{E}-04$ & 1.992 \\
1800 & 640 & $9.563667 \mathrm{E}-05$ & 2.002 & $5.625294 \mathrm{E}-05$ & 2.020 \\
3600 & 2560 & $2.385950 \mathrm{E}-05$ & 2.002 & $1.395642 \mathrm{E}-05$ & 2.011 \\
\hline \hline
\end{tabular}

Table B6. $E_{\infty}$ and $E_{2}$ for the RBF approximation of an American put with strike $K=1$ and maturity $T=1$ under a CGMY model with parameters $C=0.42, G=4.37, M=191.2$, and $Y=1.0102$, and with $r=0.06$ and $q=0$. The parameters were taken from (Wang et al., 2007). 


\begin{tabular}{cccccc}
\hline \hline$N$ & $M_{0}$ & $E_{\infty}\left(N, M_{0}\right)$ & $R_{\infty}$ & $E_{2}\left(N, M_{0}\right)$ & $R_{2}$ \\
\hline \hline 225 & 10 & $2.499711 \mathrm{E}-04$ & N/A & $1.504821 \mathrm{E}-04$ & N/A \\
450 & 40 & $6.249821 \mathrm{E}-05$ & 2.000 & $2.007511 \mathrm{E}-05$ & 2.906 \\
900 & 160 & $1.562534 \mathrm{E}-05$ & 2.000 & $5.060301 \mathrm{E}-06$ & 1.988 \\
1800 & 640 & $3.906215 \mathrm{E}-06$ & 2.000 & $1.307000 \mathrm{E}-06$ & 1.953 \\
3600 & 2560 & $9.765537 \mathrm{E}-07$ & 2.000 & $3.292508 \mathrm{E}-07$ & 1.989 \\
\hline \hline
\end{tabular}

Table B7. $E_{\infty}$ and $E_{2}$ for the RBF approximation of an American put with strike $K=1$ and maturity $T=0.25$ under a CGMY model with parameters $C=1, G=8.8, M=9.2$ and $Y=1.6$ and with $r=0.01$ and $q=0$. The parameters are the ones of Table B4, except that we now deleted the Brownian component, taking $\sigma=0$. Note again that we are in the infinite variation case: $Y>1$.

\begin{tabular}{cccccc}
\hline \hline$N$ & $M_{0}$ & $E_{\infty}\left(N, M_{0}\right)$ & $R_{\infty}$ & $E_{2}\left(N, M_{0}\right)$ & $R_{2}$ \\
\hline \hline 225 & 10 & $2.752021 \mathrm{E}-03$ & N/A & $7.132270 \mathrm{E}-04$ & N/A \\
450 & 40 & $3.330911 \mathrm{E}-04$ & 3.047 & $8.718033 \mathrm{E}-05$ & 3.032 \\
900 & 160 & $8.131372 \mathrm{E}-05$ & 2.034 & $2.160410 \mathrm{E}-05$ & 2.013 \\
1800 & 640 & $2.013791 \mathrm{E}-05$ & 2.014 & $6.101223 \mathrm{E}-06$ & 1.824 \\
3600 & 2560 & $5.027503 \mathrm{E}-06$ & 2.002 & $1.515820 \mathrm{E}-06$ & 2.009 \\
\hline \hline
\end{tabular}

Table B8. $E_{\infty}$ and $E_{2}$ for the RBF approximation of an American put with strike $K=1$ and maturity $T=0.5$ under a VG model with CGMY parameters $C=5.9311, G=20.2648, M=39.783$ and $Y=0$, and with $r=0$ and $q=0.03$. The parameters were taken from (Wang et al., 2007), except for $q=0.03$.

\begin{tabular}{cccccc}
\hline \hline$N$ & $M_{0}$ & $E_{\infty}\left(N, M_{0}\right)$ & $R_{\infty}$ & $E_{2}\left(N, M_{0}\right)$ & $R_{2}$ \\
\hline \hline 225 & 10 & $3.345919 \mathrm{E}-03$ & N/A & $1.666437 \mathrm{E}-03$ & N/A \\
450 & 40 & $1.117774 \mathrm{E}-03$ & 1.582 & $3.950589 \mathrm{E}-04$ & 2.077 \\
900 & 160 & $3.259684 \mathrm{E}-04$ & 1.778 & $9.872639 \mathrm{E}-05$ & 2.001 \\
1800 & 640 & $9.161985 \mathrm{E}-05$ & 1.831 & $2.582561 \mathrm{E}-05$ & 1.935 \\
3600 & 2560 & $2.436247 \mathrm{E}-05$ & 1.911 & $6.451929 \mathrm{E}-06$ & 2.001 \\
\hline \hline
\end{tabular}

Table B9. $\quad E_{\infty}$ and $E_{2}$ for the RBF approximation of an American put with strike $K=1$ and maturity $T=0.25$ under a VG model with CGMY parameters $C=1.25, G=2.73, M=5.73$ and $Y=0$, and with $r=0.1$ and $q=0$. The parameters are taken from (Matache et al., 2005a).

\begin{tabular}{cccccc}
\hline \hline $\mathrm{N}$ & $M_{0}$ & $E_{\infty}\left(\hat{x}_{i}, T\right)$ & $R_{\infty}$ & $E_{2}\left(\hat{x}_{i}, T\right)$ & $R_{2}$ \\
\hline \hline 3600 & 10 & $5.695862 \mathrm{E}-04$ & $\mathrm{~N} / \mathrm{A}$ & $2.267973 \mathrm{E}-04$ & N/A \\
3600 & 40 & $5.759096 \mathrm{E}-05$ & 1.653 & $2.113057 \mathrm{E}-05$ & 1.712 \\
3600 & 160 & $1.478201 \mathrm{E}-05$ & 0.981 & $5.275323 \mathrm{E}-06$ & 1.001 \\
3600 & 640 & $3.911633 \mathrm{E}-06$ & 0.959 & $1.339096 \mathrm{E}-06$ & 0.989 \\
3600 & 2560 & $9.765537 \mathrm{E}-07$ & 1.001 & $3.292508 \mathrm{E}-07$ & 1.012 \\
\hline
\end{tabular}

Table B10. $E_{1}$ and $E_{2}$ for the American put of Table B7. We fixed the number of the interpolation points is fixed to 3600 and let the number of time steps $M_{0}$ increase. The results indicate first order of convergence in time. 

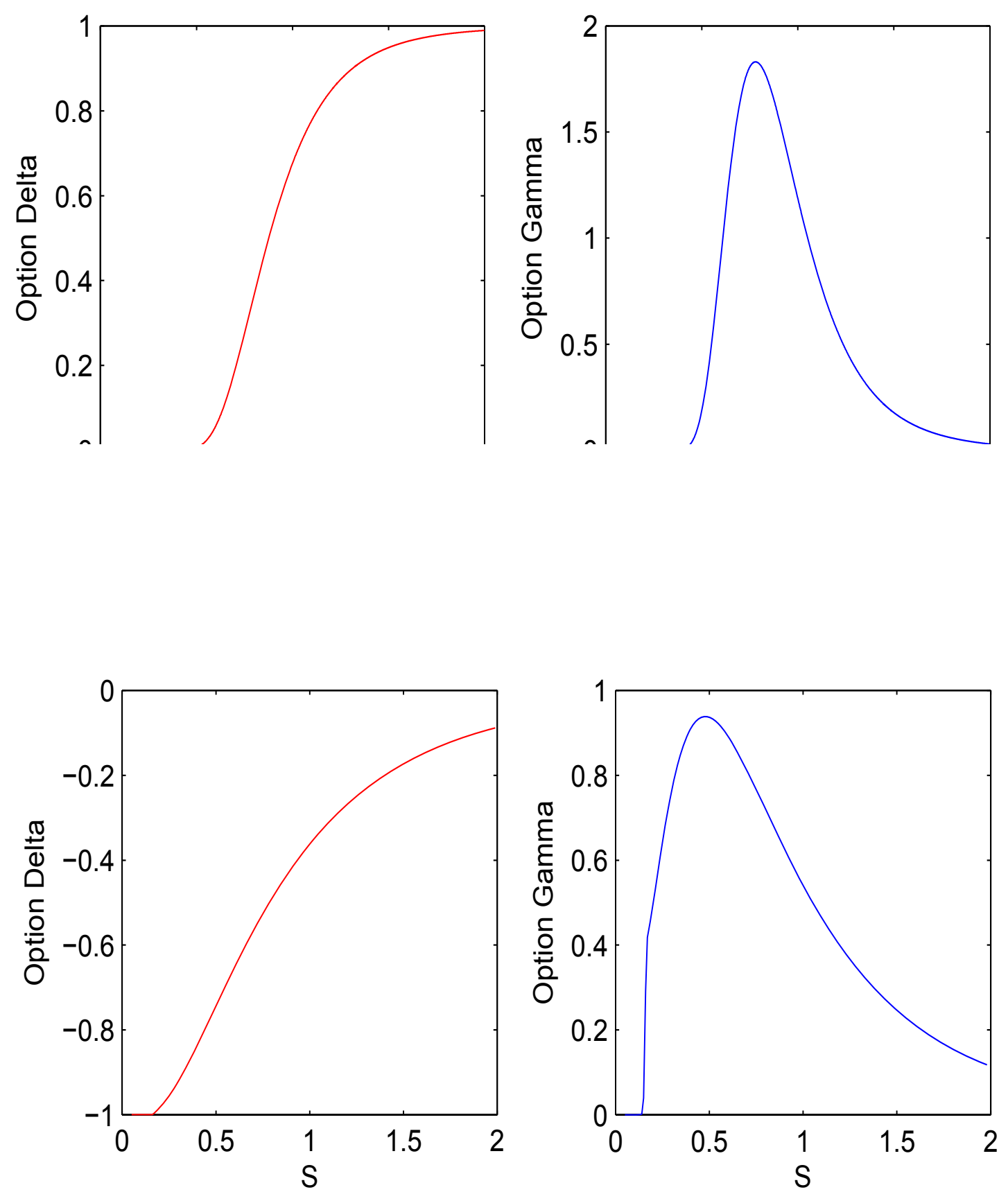

Figure B2. $\Delta$ (Left) and $\Gamma$ (Right) for the American put option of table B7 with $Y=1.6$. Note that since the underlying process has infinite variation, smooth pasting holds. This is also apparent from the bottom left part of the graph for $\Delta$. The number of the interpolation points used was 3600 .

(1) The 

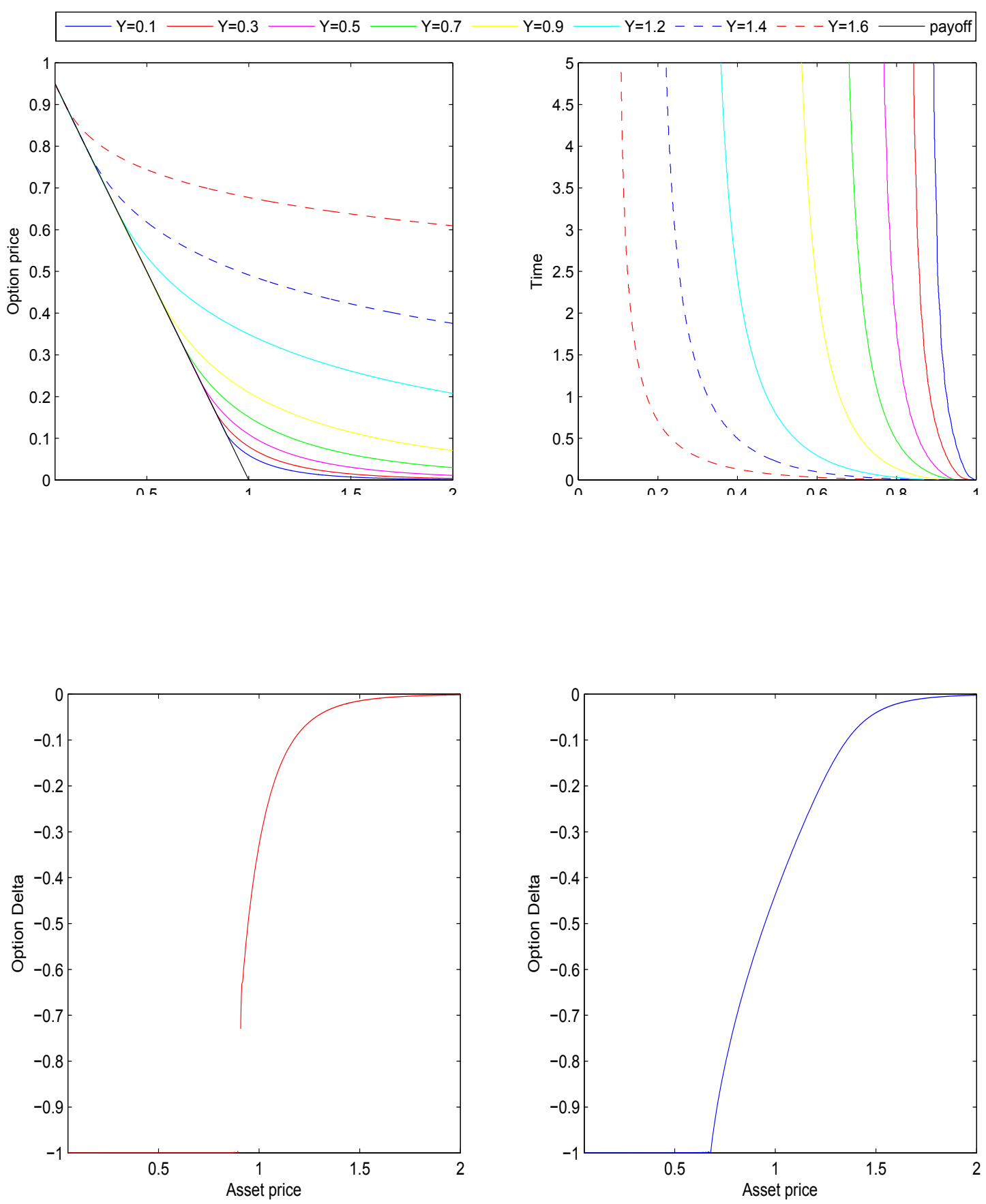

Figure B4. Left picture: Discontinuous option Delta for $G=7$ and $M=9$. Right picture: Continuous option Delta for $G=10$ and $M=3 ; \sigma=0, r=0.1, q=0, Y=0.2, C=1, K=1$ and $T=1$. Parameters as in (Almendral, 2004), except for $K=1$. 

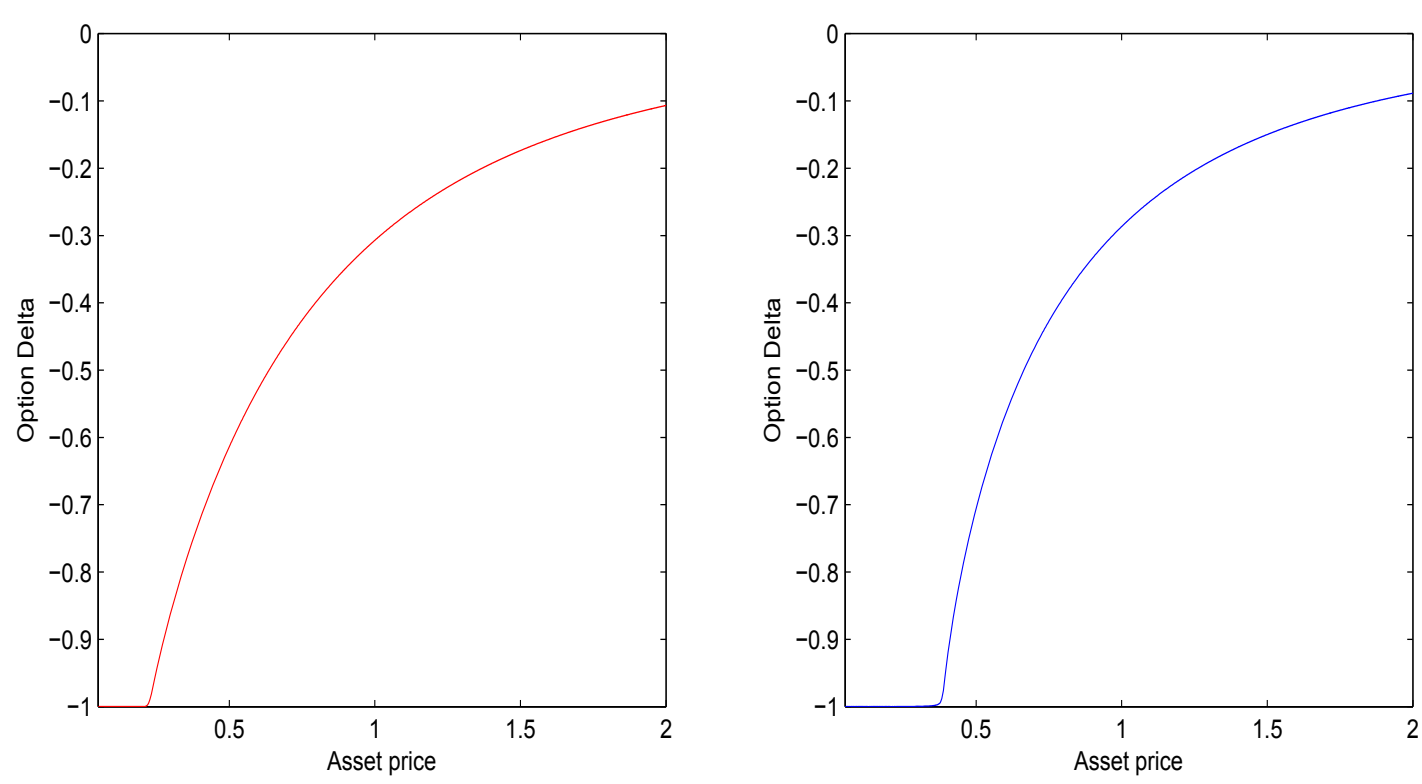

Figure B5. Illustration of smooth fit for two infinite variation CGMY processes $(Y>1$.) Left picture: $r=0.1, q=0$, $\sigma=0, C=1, G=8.8, M=9.2, Y=1.6, K=1$, and $T=0.5$. Right picture: $r=0.4, q=0, \sigma=0.2, C=1, G=1.4$,

$M=2.5, Y=1.4, K=1$, and $T=0.5$. Except $K=1$, all parameters are copied from (Matache et al., 2005a). 